\title{
On defining progress variable for Raman/Rayleigh experiments in partially-premixed methane flames
}

\author{
Robert. S. Barlow ${ }^{1 *}$, Gaetano Magnotti ${ }^{1}$, Hugh C. Cutcher ${ }^{2}$, Assaad R. Masri $^{2}$ \\ ${ }^{1}$ Combustion Research Facility, Sandia National Laboratories, Livermore, CA 94566 \\ ${ }^{2}$ School of Aerospace, Mechanical and Mechatronic Engineering, \\ The University of Sydney, Sydney 2006 NSW, Australia
}

\begin{abstract}
Progress variable is a central concept in the theory and modeling of partially-premixed flames. However, there has been no consensus in the literature on its definition, particularly in the context of experiments. Several possible definitions of progress variable are considered, based on mass fractions of the seven major species that can be measured by combined Raman/Rayleigh scattering methods applied to turbulent methane flames. The behavior of three candidate progress variables is evaluated using laminar opposed-flow flame calculations. The effects of normalizing by the fully-burnt state rather than the more commonly used equilibrium state are considered. A progress variable, $c_{O}$, defined as the mass of oxygen bound in the products $\mathrm{CO}_{2}, \mathrm{CO}$ and $\mathrm{H}_{2} \mathrm{O}$, divided by that which would be bound if the sample were taken to its fully-burnt state, is argued to offer some advantages over the other definitions considered. It is shown that inclusion of a larger number of species in calculating $c_{O}$ (18 versus 7) makes little difference, such that the seven major species accessible to Raman/Rayleigh laser diagnostics are sufficient to quantify reaction progress in partially-premixed methane flames at atmospheric pressure. The measured joint statistics of mixture fraction, $Z$, and progress variable, $c_{O}$, are presented for two turbulent piloted partially-premixed methane-air jet flames that have significantly different mixture fraction profiles at the jet exit. Favre average radial profiles of first and second moments, as well as the correlation coefficient, $R_{Z, c}$, highlight differences in near-field scalar structure between the two flames. Examples of joint $Z, c_{O}$ histograms, sampled from $0.3-\mathrm{mm}$ segments centered at the radial location where $\tilde{Z}=0.065$ in each profile, are
\end{abstract}


presented. This mixture fraction value corresponds roughly to the location of peak heat release rate on the fuel-rich side in the laminar flame calculations. A clear conclusion is that the common modeling assumption of statistical independence of mixture fraction and progress variable is contradicted by the turbulent flame measurements. The proposed progress variable can be easily calculated from simulations as a post-processing step, independent of the modeling approach, allowing for consistent comparison of measured and modeled turbulent flame results.

Keywords: Progress variable; mixture fraction; turbulent flames; partially-premixed flames

Corresponding author:

E-mail address: barlow@ sandia.gov (Robert S. Barlow)

Phone: +1-925-294-2688 


\section{Introduction}

Mixture fraction, $Z$, and progress variable, $c$, are central concepts in turbulent combustion theory and in flamelet modeling of turbulent partially-premixed flames [1-11]. It is common in both experimental and modeling studies to use Bilger's formula for mixture fraction [12], which preserves its stoichiometric value in the presence of differential species diffusion. This property is desirable in the context of Raman/Rayleigh experiments because differential diffusion effects are not negligible in typical laboratory-scale turbulent flames, especially within the near field of flames operated at low to moderate Reynolds number [13-15]. There is not such a consensus in the combustion literature regarding the definition of progress variable for partially-premixed flames. In flamelet modeling approaches with tabulated chemistry, it is common to define progress variable as a sum of major product mass fractions, often without normalization. Examples for hydrocarbon flames include $Y_{\mathrm{CO} 2}$ [16], $Y_{\mathrm{CO} 2}+Y_{\mathrm{H} 2 \mathrm{O}}[3], Y_{\mathrm{CO} 2}+Y_{\mathrm{CO}}[2,4,6,11,17]$, $Y_{\mathrm{CO} 2}+Y_{\mathrm{CO}}+Y_{\mathrm{H} 2 \mathrm{O}}$ [18], and $Y_{\mathrm{CO} 2}+Y_{\mathrm{CO}}+Y_{\mathrm{H} 2 \mathrm{O}}+Y_{\mathrm{H} 2}$ [19]. Progress variable has also been defined on a molar basis as $Y_{\mathrm{CO} 2} / w_{\mathrm{CO} 2}+Y_{\mathrm{H} 2 \mathrm{O}} / w_{\mathrm{H} 2 \mathrm{O}}+Y_{\mathrm{H} 2} / w_{\mathrm{H} 2}$ [7,20,21]. More recently, various 'optimized' progress variables have been proposed [22-25] that allow for improved efficiency and accuracy in storage and retrieval of thermochemical states from flamelet tables. These optimized progress variables can be linear combinations of many species, including minor species that are not experimentally accessible. Such progress variables offer computational benefits. However, they serve primarily as tabulation parameters, are usually too complex to be used for experiments, and do not necessarily conform to the original theoretical concept of a normalized variable that quantifies chemical progress from reactants toward products. Even more recently, it has been suggested [26] that weighing factors for experimentally accessible species can be optimized to minimize uncertainty in the experimentally derived progress variable. Such an approach is beyond the scope of the present work but is a promising option for future research.

Experimental results on progress variable in partially-premixed flames are rare in the literature [9,27,28]. Measurements of progress variable and its dissipation rate have been reported for premixed and stratified flames [27-30], with $c_{T}=\left(T-T_{0}\right) /\left(T_{e q}(Z)-T_{0}\right)$, where $T_{0}$ is the unburnt reactant temperature and $T_{e q}(Z)$ is the adiabatic equilibrium temperature for the local value of mixture fraction. In Raman/Rayleigh experiments in stratified flames the selection of the temperature-based progress variable was motivated by the higher precision of temperature 
measurements, which are derived primarily from Rayleigh scattering signals, compared to species measurements, which are derived primarily from the weaker Raman scattering signals. Experimental noise is of particular concern for measurements of gradients and dissipation because gradient operators amplify relative noise in a signal. However, a temperature-based progress variable is only appropriate for flames with negligible heat loss, and it is not favored in modeling. Vreman et al. [7] compared flamelet-based simulations of progress variable in Sandia flame D with results calculated from the measured mass fractions of major species. Parente et al. [31] applied principle component analysis to represent thermochemical states from turbulent flame measurements using a reduced number of scalars, offering an alternative "optimized" method for analyzing and comparing experimental and computational results. Conditional means and conditional pdfs of "reactedness" or "burning index" have been reported for nonpremixed and partially-premixed jet flames as indicators of the probability of local extinction $[32,33]$. These have been defined similarly to progress variable, using temperature or single species, for the purpose of comparing measured and modeled results.

The objective of the present study is to develop a species-based definition of progress variable that can be used easily in the context of Raman/Rayleigh experiments in partiallypremixed methane flames and can allow for direct comparisons of experimental and computational results for such things as joint and conditional statistics of mixture fraction and progress variable, as well as gradients and dissipation rates of these quantities. Raman/Rayleigh experiments in methane-air flames yield temperature and the mass fractions of major species $\left(\mathrm{N}_{2}\right.$, $\mathrm{O}_{2}, \mathrm{CH}_{4}, \mathrm{CO}_{2}, \mathrm{H}_{2} \mathrm{O}, \mathrm{CO}$, and $\mathrm{H}_{2}$ ). Therefore, an experimentally compatible progress variable should be calculated from mass fractions of a subset of these seven species. Furthermore, it seems preferable to define a normalized progress variable and to use a reference condition for normalization that does not depend on assumptions regarding molecular transport. Specifically, the definition should not depend on an assumption of equal diffusivities. This means that the reference state for normalization should be calculated directly from properties of each measured sample (e.g., a 'fully-burnt' state to be define below), not from an equilibrium table or an assumed laminar flame solution.

Normalizing the progress variable by equilibrium, by the fully-burnt reference states, or by an assumed laminar flame solution introduces a dependence on mixture fraction. As a consequence, extra terms appear in the transport equation for the progress variable $[1,5,6,15]$ 
which require additional modeling. The inclusion of these extra terms may be considered a disadvantage by some. However, we do not suggest that the definition of progress variable developed here need be used directly in models. For the purpose of comparison, the proposed progress variable could be calculated in a post-processing step, independent of the modeling approach.

In the following sections we consider alternative definitions of progress variable based on mass fractions of the measured major species in methane flames. The behavior of these progress variables is examined using both laminar flame calculations and measurements from turbulent piloted partially-premixed methane-air jet flames [34-36]. The effects of including a larger number of species in calculating progress variable and mixture fraction are quantified, as are the effects of using the fully-burnt state rather than equilibrium for normalization. A normalized progress variable based on the oxygen mass in the main product species $\left(\mathrm{CO}_{2}, \mathrm{H}_{2} \mathrm{O}\right.$ and $\left.\mathrm{CO}\right)$ is shown to offer some advantages and is proposed for future work. Example results on the joint statistics of mixture fraction and progress variable in two turbulent jet flames, with very different exit profiles of mixture fraction and different near-field modes of combustion, are presented and compared. These results contradict the common modeling assumption of statistical independence of progress variable and mixture fraction.

\section{Definitions of progress variable, equivalence ratio, and mixture fraction}

Three definitions of progress variable are considered here, each based on mass fractions of the seven major species measured in Raman/Rayleigh experiments in methane flames $\left(\mathrm{N}_{2}, \mathrm{O}_{2}\right.$, $\mathrm{CH}_{4}, \mathrm{CO}_{2}, \mathrm{H}_{2} \mathrm{O}, \mathrm{CO}$, and $\mathrm{H}_{2}$ ). These are:

- $c_{C}$ based on the sum of $\mathrm{CO}_{2}$ and $\mathrm{CO}$ mass fractions

- $c_{Y}$ based on the sum of $\mathrm{CO}_{2}, \mathrm{CO}, \mathrm{H}_{2} \mathrm{O}$ and $\mathrm{H}_{2}$ mass fractions

- $c_{O}$ based on the oxygen mass fraction contained in $\mathrm{CO}_{2}, \mathrm{CO}$, and $\mathrm{H}_{2} \mathrm{O}$.

The progress variable based on oxygen is easiest to calculate. It is defined as the mass of $\mathrm{O}$ in the sample that is bound to fuel atoms in the form of $\mathrm{CO}_{2}, \mathrm{CO}$, and $\mathrm{H}_{2} \mathrm{O}$ divided by the mass of $\mathrm{O}$ that could be bound if the sample were taken fully to products, $\mathrm{CO}_{2}$ and $\mathrm{H}_{2} \mathrm{O}$, consuming any deficient reactants. Thus, there are separate expressions for lean and rich samples. The 
numerator is the same in both, but the denominators are different. In lean samples there is excess oxygen, so any $\mathrm{CH}_{4}, \mathrm{CO}$, or $\mathrm{H}_{2}$ in the sample can be fully converted to $\mathrm{CO}_{2}$ and $\mathrm{H}_{2} \mathrm{O}$, while in stoichiometric or fuel-rich samples all of the available $\mathrm{O}_{2}$ can be consumed. The fully-burnt state is used as the reference state for simplicity and to avoid assumptions regarding molecular transport, which would be required if the reference state were interpolated from pre-calculated equilibrium or low-strain flamelet tables. Thus the progress variable based on oxygen is calculated as:

$$
\begin{aligned}
& c_{O}=\frac{Y_{C O 2}\left(\frac{w_{O 2}}{w_{C O 2}}\right)+Y_{C O}\left(\frac{w_{O}}{w_{C O}}\right)+Y_{H 2 O}\left(\frac{w_{O}}{w_{H 2 O}}\right)}{\left(Y_{C O 2}\left(\frac{w_{O 2}}{w_{C O 2}}\right)+Y_{C O}\left(\frac{w_{O 2}}{w_{C O}}\right)+Y_{H 2 O}\left(\frac{w_{O}}{w_{H 2 O}}\right)+Y_{H 2}\left(\frac{w_{O}}{w_{H 2}}\right)+Y_{C H 4}\left(\frac{2 w_{O 2}}{w_{C H}}\right)\right)} ; \phi<1 \\
& c_{O}=\frac{Y_{C O 2}\left(\frac{w_{O 2}}{w_{C O 2}}\right)+Y_{C O}\left(\frac{w_{O}}{w_{C O}}\right)+Y_{H 2 O}\left(\frac{w_{O}}{w_{H 2 O}}\right)}{\left(Y_{C O 2}\left(\frac{w_{O 2}}{w_{C O 2}}\right)+Y_{C O}\left(\frac{w_{O}}{w_{C O}}\right)+Y_{H 2 O}\left(\frac{w_{O}}{w_{H 2 O}}\right)+Y_{O 2}\right)} ; \phi \geq 1
\end{aligned}
$$

where $Y_{i}$ are species mass fractions and $w_{i}$ are molar masses. Here, as in our previous experimental work, the equivalence ratio is calculated as the oxygen required divided by the oxygen available, based on the mole fractions of the same seven major species. This ensures consistency in the application of Eqn. (1) to experimental results.

$$
\phi=\frac{X_{\mathrm{CO}_{2}}+2 X_{\mathrm{CH}_{4}}+X_{\mathrm{CO}}+0.5\left(X_{\mathrm{H}_{2} \mathrm{O}}+X_{\mathrm{H}_{2}}\right)}{X_{\mathrm{CO}_{2}}+X_{\mathrm{O}_{2}}+0.5\left(X_{\mathrm{CO}}+X_{\mathrm{H}_{2} \mathrm{O}}\right)}
$$

Progress variables $c_{C}$ and $c_{Y}$ are similarly defined, using the fully-burnt state of the sample for normalization. For example, $c_{C}$ is defined as the sum of $\mathrm{CO}_{2}$ and $\mathrm{CO}$ mass fractions in the sample divided by the sum of $\mathrm{CO}_{2}$ and $\mathrm{CO}$ mass fractions that would be present if all deficient reactants were converted to products. However, with differential diffusion and also due to experimental noise, the local $\mathrm{C} / \mathrm{H}$ ratio may differ from 0.25 , so additional tests and rules must be applied in calculating the reference state for rich samples. Complete expressions for $c_{C}$ and $c_{Y}$ are somewhat cumbersome and are given in the supplemental Appendix.

Mixture fraction is calculated from the measured major species, using Bilger's definition,

$$
Z=\frac{2\left(Y_{C}-Y_{C, 2}\right) / w_{C}+\left(Y_{H}-Y_{H, 2}\right) / 2 w_{H}-\left(Y_{O}-Y_{O, 2}\right) / w_{O}}{2\left(Y_{C, 1}-Y_{C, 2}\right) / w_{C}+\left(Y_{H, 1}-Y_{H, 2}\right) / 2 w_{H}-\left(Y_{O, 1}-Y_{O, 2}\right) / w_{O}}
$$


where $Y_{i}$ are elemental mass fractions, and the subscripts 1 and 2 refer to conditions in the rich (fuel) and lean (oxidizer) streams, respectively [12].

\section{Behavior in laminar flame calculations}

In this section the results of laminar opposed-flow simulations from Chemkin Pro [37], with GRI Mech 3.0 [38] and multi-component transport with the Soret effect included, are used to examine the behavior of the three progress variables, $c_{O}, c_{C}$, and $c_{Y}$, in strained partiallypremixed flames.

Figure 1 shows that the three progress variables have similar behavior over a range of richside equivalence ratios $\left(\phi_{\mathrm{r}}=1.3,1.7,3.2,4.8,9.5\right)$ and for pure $\mathrm{CH}_{4}$ on the fuel side. These calculations were run at two values of the nominal strain parameter, $a=100 \mathrm{~s}^{-1}$ and $a=300 \mathrm{~s}^{-1}$. Air was represented by $\mathrm{N}_{2}$ and $\mathrm{O}_{2}$ only. The smallest differences are seen with $\phi_{\mathrm{r}}=1.3$ at the lower strain rate (Fig. 1a), and the differences among the three progress variables increase with increasing fuel-side equivalence ratio and increasing strain. This is a combined effect of differential diffusion and finite rate chemistry. For example, the strain rate on the flame in Fig. 1a is low enough to allow a true double flame, with a rich premixed reaction zone that is well separated from the stoichiometric diffusion flame, such that the progress variables nearly reach unity before the effects of diffusive coupling cause mixture fraction to decrease from the richside boundary value. Also, at low to moderate strain rate there is little leakage of $\mathrm{CO}$ or $\mathrm{H}_{2}$ past the lean side of the reaction zone, so all progress variables are close to unity in cooler lean mixtures. Differential diffusion causes the $\mathrm{C} / \mathrm{H}$ atom ratio to decrease toward the air boundary, so values of the three progress variables in that region go as $c_{C}<c_{O}<c_{Y}$, but the differences are minor.

In contrast, the pure diffusion flame at $a=300 \mathrm{~s}^{-1}$ (Fig. $1 \mathrm{j}$ ), which is not far from the extinction limit, shows greater differences among the progress variables. Here, there is greater leakage of $\mathrm{O}_{2}$ past rich side of the reaction zone, due to finite rate chemistry. This oxygen diffuses toward the rich boundary along with products, such that the normalized progress variables remain nearly constant across rich mixtures, but at a lower level than in the pure diffusion flame at $a=100 \mathrm{~s}^{-1}$ strain rate. At the higher strain rate there is also greater leakage of 
$\mathrm{CO}$ and $\mathrm{H}_{2}$ past the lean side of the reaction zone, so the progress variable curves in that region are all shifted downward. Furthermore, because $\mathrm{H}_{2} \mathrm{O}$ production continues to a lower temperature than $\mathrm{CO}_{2}$ production, the increased strain rate allows relatively more $\mathrm{CO}$ than $\mathrm{H}_{2}$ to penetrate toward the air side, and this contributes to greater differences among the three normalized progress variables. For all of the flames in Fig. 1., $c_{O}$ and $c_{Y}$ have similar values near the air side, with $c_{C}$ taking lower values, while $c_{O}$ and $c_{C}$ are have similar values approaching stoichiometric (vertical dashed line) from the lean side, with $c_{Y}$ taking higher values. Moving into rich mixtures, $c_{C}$ increases more rapidly to slightly exceed $c_{Y}$ at the local rich-side maximum (or plateau in the pure diffusion flames). In summary, there are differences among the three progress variables in these opposed laminar flames with multi-component transport, but the differences are relatively small.

Normalized progress variables for partially-premixed combustion have rarely, if ever, been plotted in literature, so the behavior shown in Fig. 1 may be unfamiliar. All three progress variables have a local minimum or "dip" near the stoichiometric condition, which can seem counterintuitive. However, this behavior is easily explained and is a physically correct reflection of the relative progress of reaction from reactants to products for the local thermochemical state. There is also a discontinuity in slope at the stoichiometric mixture fraction, and this is a consequence of using the fully-burnt state for normalization because the denominators in Eqns. (1a) and (1b) have different slopes at stoichiometric.

Both issues are clarified by Fig. 2a, which shows results for the oxygen-based progress variable, $c_{O}$, and the corresponding the non-normalized progress variable, $Y_{O, b}$, the mass of oxygen bound in $\mathrm{CO}_{2}, \mathrm{CO}$, and $\mathrm{H}_{2} \mathrm{O}$, in a strained flame calculation with all diffusivities equal $(\mathrm{Le}=1)$. The progress variable $c_{O}$ has been calculated two ways, first as in Eqn. (1), using the fully-burnt state for normalization, and second using the equilibrium state for normalization. There is little difference between the FB (fully-burnt) and EQ (equilibrium) curves for $c_{O}$, so it is clear that the dip is not attributable to the use of fully-burnt versus equilibrium for normalization. It is well known that there is overlap of fuel and oxidizer in any diffusion flame, and the degree of overlap increases with strain rate. In Fig. 2a there are three curves for $Y_{O, b}$, each calculated from the experimentally accessible major species. The dash-dot (blue) line is from the flame solution; the dash (red) line is from equilibrium; and the solid (black) line is the fully-burnt state 
calculated from the flame solution according to the denominators in Eqns. (1a) and (1b). A scaling factor of two has been applied to better reveal the deficit in the $Y_{O, b}$ curve for the flame near the stoichiometric condition. This deficit between the flame solution dash-dot (blue) and the two other curves around stoichiometric results from finite rate chemistry, and it translates to the dip in the normalized progress variable.

Figures $2 \mathrm{~b}$ and $2 \mathrm{c}$ show the corresponding non-normalized and normalized results for progress variables based on the sums $Y_{\mathrm{CO} 2}+Y_{\mathrm{CO}}+Y_{\mathrm{H} 2 \mathrm{O}}+Y_{\mathrm{H} 2}$ and $Y_{\mathrm{CO} 2}+Y_{\mathrm{CO}}$, respectively. Behavior for lean and near-stoichiometric conditions is similar to that for the bound-oxygen progress variable. Due to finite rate chemistry, slow $\mathrm{CO}$ chemistry in particular, fuel $\left(\mathrm{CO}\right.$ and $\left.\mathrm{H}_{2}\right)$ coexist with $\mathrm{O}_{2}$ in the region near stoichiometric, so the product mass fractions are lower than in the equilibrium or fully-burnt states. The three normalized progress variables all correctly reflect this aspect of strained flame structure.

There are significant differences between the equilibrium and fully-burnt curves in Figs. 2b and 2c. This is due to the fact the equilibrium states for methane-air mixtures richer than about $\phi=1.3$ have CO levels much higher than would ever occur in a realizable laminar or turbulent flame. At the fuel-rich boundary condition, which corresponds to a 3:1 air to methane ratio, the equilibrium mass fractions of the four product species are $Y_{\mathrm{CO}}=0.2250, Y_{\mathrm{CO} 2}=0.0553$, $Y_{\mathrm{H} 2}=0.0340$, and $Y_{\mathrm{H} 2 \mathrm{O}}=0.0312$. The available oxygen is dominantly claimed by carbon. Consequently, the mass fraction sums in Figs. $2 b$ and $2 c$ are much higher in rich mixtures than the sums obtained by taking the flame results to a fully-burnt state according to the equations in the supplemental Appendix. The fully-burnt product mass fractions at the fuel-rich boundary are $Y_{\mathrm{CO} 2}=0.1351, Y_{\mathrm{H} 2 \mathrm{O}}=0.1106, Y_{\mathrm{CO}}=0.0$, and $Y_{\mathrm{H} 2}=0.0$.

For such rich mixtures it can reasonably be argued that equilibrium is not an appropriate reference state for the two progress variables based directly on product mass fractions. By doing the bookkeeping according to the mass of oxygen bound in the same products, the rich-side differences are eliminated between equilibrium and the calculated fully-burnt mass fractions, $Y_{O, b}$, so that the two curve for $c_{O}$ (normalized by fully-burnt versus equilibrium) differ only in the region near stoichiometric. Note also that $c_{O}$ will take the same value whether calculated on the basis of mass fractions or mole fractions because the molar mass of oxygen cancels out. 
Figure 3 illustrates the problems associated with using an equilibrium table for normalization of a progress variable in a flame where differential diffusion is important. The flame is the same as for Fig. $1 \mathrm{~b}\left(\phi_{\mathrm{r}}=3.2, a=100 \mathrm{~s}^{-1}\right.$, multi-component transport, Soret $)$, but the three progress variables were calculated using the equilibrium state rather than the fully-burnt state for normalization. There are significant differences among the three alternatively defined progress variables, not only in fuel-rich conditions but throughout the mixture fraction domain. The curves diverge rapidly near the air boundary, where differential diffusion causes the $\mathrm{C} / \mathrm{H}$-atom ratio to decrease toward zero.

There are some clear benefits to using the fully-burnt state for normalization. However, the fact that normalization by the fully-burnt state creates a cusp at the stoichiometric mixture fraction, whereas equilibrium yields a smooth curve, is awkward in the context of gradient and dissipation measurements in turbulent flames. Gradients are often calculated by central differencing, which acts as a spatial filter. However, instantaneous profiles of the progress variable gradient could still abruptly change sign. One might expect artificially high values of the conditional mean dissipation of progress variable near stoichiometric, and the conditional mean cross dissipation could also abruptly change sign. These potential artifacts can be avoided by applying a simple correction or renormalization of the oxygen-based progress variable that is illustrated in Fig. 4. The dashed (red) curve in Fig. 4 is obtained by applying Eqn. (1) to the tabulated equilibrium states. This is the progress variable (labeled $c_{O, e q}$ ) comparing equilibrium to the fully-burnt reference state. $c_{O, e q}$ is unity $(>0.999)$ everywhere except within the region close to stoichiometric, where the minimum value is 0.971 . The two solid (black) curves show progress variable $c_{O}$ for the flames with $\phi_{\mathrm{r}}=3.2$ and $a=100$ and $300 \mathrm{~s}^{-1}$ (Figs. $1 \mathrm{~b}$ and $1 \mathrm{~g}$, including multi-component transport). The dash-dot (blue) curves are obtained as $c_{O} / c_{O, e q}$ and form smooth bridges across the stoichiometric region. This approximates the result one would get by using the local equilibrium state for normalization without having to calculate that state for each point along the multi-component-transport laminar flame solution. Magnitudes are changed little, but the behavior of the gradient is much improved.

It is proposed that the correction or renormalization described above be included in the progress variable definition for application to experimental data and for comparison with simulations. It is important to point out that the correction should be tabulated and interpolated 
as a function of equivalence ratio, calculated as in Eqn. (2), rather than mixture fraction, in order to ensure consistent alignment and a smooth bridge. This is because the value of mixture fraction at the stoichiometric condition defined by Eqn. (2) depends on the boundary conditions used in Eqn. (3), which may include ambient humidity and $\mathrm{CO}_{2}$, for example. Note also that the simple relationship between mixture fraction and equivalence ratio given in Eqn. (4), where $Z_{s t}$ is the stoichiometric value of mixture fraction, only strictly applies if mass diffusivities are equal and if ambient water vapor $\left(\sim 0.01\right.$ mole fraction in the experiments) and $\mathrm{CO}_{2}$ are neglected in calculating the mixture fraction.

$$
\phi \approx \frac{Z}{1-Z} \frac{1-Z_{s t}}{Z_{s t}}
$$

This expression should not be used to determine equivalence ratio for the test applied in calculating the normalization reference state as in Eqn. (1).

It is important to consider what limitations are imposed by the fact that only seven major species are available from most Raman/Rayleigh experiments in methane flames. As a test, the following additional species were included from the Chemkin laminar flame simulations: $\mathrm{OH}, \mathrm{H}$, $\mathrm{O}, \mathrm{CH}_{3}, \mathrm{CH}_{2} \mathrm{O}, \mathrm{C}_{2} \mathrm{H}_{2}, \mathrm{C}_{2} \mathrm{H}_{4}, \mathrm{C}_{2} \mathrm{H}_{6}, \mathrm{CH}_{3} \mathrm{OH}, \mathrm{HCCO}$, and $\mathrm{CH}_{2} \mathrm{CO}$, giving a total of 18 species. Extended versions of Eqns. (1-3) were applied in calculating mixture fraction, equivalence ratio, and progress variable. Figure 5 compares results based on 18 versus 7 species in two partiallypremixed flames $\left(\phi_{\mathrm{r}}=1.3,3.2\right)$ at $a=100 \mathrm{~s}^{-1}$ and a pure diffusion flame at $a=300 \mathrm{~s}^{-1}$. These flames correspond to Figs. 1a, 1b, and 1j. The superscript asterisk indicates progress variable, $c_{O}^{*}$, and mixture fraction, $Z^{*}$, determined from 18 species. In each frame, $c_{O}$ is plotted versus $Z$, $c_{O}^{*}$ is plotted versus $Z^{*}$, and the ratios $c_{O}^{*} / c_{O}$ and $Z^{*} / Z$ evaluated at each point along the Chemkin solution are plotted versus $Z$, the 7-species mixture fraction. While there are apparent differences in the curves for progress variable, it is only the mixture fraction values that are affected significantly by including more species. The effect on the calculated progress variable never exceeds 1 percent, while maximum values of $Z^{*} / Z$ range from 1.065 on the rich side of the double flame (Fig. 5a) to 1.092 in the pure diffusion flame (Fig. 5c).

It should be emphasized that most of the additional hydrocarbon species contribute Raman scattering signals in the same $\mathrm{C}-\mathrm{H}$ stretch region of the spectrum that is used to measure $\mathrm{CH}_{4}$, and the methods of calibration and data processing in typical Raman/Rayleigh experiments are such that the mass fraction assigned to $\mathrm{CH}_{4}$ approximates the total hydrocarbon mass fraction 
[39]. Therefore, the systematic error in the measured mixture fraction is expected to be less than indicated by the present laminar flame analysis. This will be examined in greater detail in future experiments on laminar opposed-flow methane/air flames, taking advantage of recent work to develop empirically based Raman spectral libraries for several C2 hydrocarbons [40,41].

The internal structure of the opposed flow laminar flames from Fig. 1 is examined further in Fig. 6, which includes spatial profiles of temperature, as well as temperature, heat release rate, and progress variable plotted against mixture fraction. Here the 18 -species versions, $c_{O}^{*}$ and $Z^{*}$, are shown. The two symbols along each profile mark the lean and rich local maxima in volumetric heat release rate. As mentioned above, the flame with $\phi_{\mathrm{r}}=1.3$ and $a=100 \mathrm{~s}^{-1}$ is a true double flame with a rich premixed flame that is well separated spatially from the stoichiometric location. The heat release curve for this rich premixed flame forms a narrow spike and drops to near zero before mixture fraction begins to decrease due to coupling with the diffusion flame. Correspondingly, the progress variable increases from zero to roughly 0.98 before mixture fraction begins to decrease significantly due to diffusion.

When the strain rate on this $\phi_{\mathrm{r}}=1.3$ flame is increased to $a=300 \mathrm{~s}^{-1}$, the rich side peak heat release shifts from $Z^{*}=0.0691$ to $Z^{*}=0.0625$, so there is clearly some coupling of the rich reaction zone with the diffusion flame. The width in mixture fraction of the rich-side peak in heat release remains relatively narrow compared to that in the pure diffusion flame, and the trajectory of progress variable leading up to the condition of peak heat release is near vertical. The rich-side peak in heat release in the $\phi_{\mathrm{r}}=1.7, a=100 \mathrm{~s}^{-1}$ flame is already shifted in mixture fraction from the boundary value. Generally, the rich-side peak in heat release moves to lower mixture fraction with increasing strain and increasing fuel-side equivalence ratio, and the width of the rich-side heat release curve broadens and extends into lean mixtures. However, there is nothing obvious in the heat release curves to distinguish the structure of the partially-premixed flames with $\phi_{\mathrm{r}}>1.7$ from that of the pure diffusion flame at the same strain rate.

An interesting behavior revealed in Fig. 6 is that, for a given strain rate, the value of the peak rich-side heat release rate and the value of progress variable at the location of peak heat release each have a non-monotonic dependence on fuel-side equivalence ratio. Peak heat release rate first decreases with increasing $\phi_{\mathrm{r}}$ then increases, and it is lowest for $\phi_{\mathrm{r}}=2.1$ at both strain rates. Progress variable at the location of peak heat release first increases with increasing $\phi_{\mathrm{r}}$ then 
decreases. The highest rich-side values of progress variable for the flames considered in Fig. 6 occur in the $\phi_{\mathrm{r}}=3.2$ flame for the lower strain rate and in the $\phi_{\mathrm{r}}=2.1$ flame for the higher strain rate. An additional observation is that, for $\phi_{\mathrm{r}}=1.3,2.1$, and 3.2 at both strain rates, peak heat release occurs at locations in the flame where the progress variable is increasing with decreasing mixture fraction ( $d c / d Z$ is negative). Further increase in $\phi_{\mathrm{r}}$ causes the location of peak heat release to move into the region of the dip in progress variable, such that the local gradient, $d c / d Z$, becomes positive. One might speculate that this behavior is related to the transition from premixed to diffusion flame structure on the fuel-rich side of these partially-premixed flames. Identification of experimentally based regime indicators is an important area for further work.

\section{Turbulent flames results}

The turbulent flames considered here are the Sydney piloted, partially-premixed $\mathrm{CH}_{4} /$ air jet flames [34-36]. These flames are stabilized on a modified burner (see Fig. 7) that includes a retractable central tube within the main jet tube, such that fuel and air can be delivered separately. The degree of fuel-air mixing depends on the recess distance $L_{\mathrm{r}}$ of the central tube and on the configuration, either fuel in the central jet (FJ) with air through the annulus or fuel in the annulus (FA) with air through the central tube. For a large recess distance $\left(L_{\mathrm{r}}=300 \mathrm{~mm}\right)$ the fuel-air mixture at the burner exit is nearly homogeneous for either configuration, and the stability of the flame, as measured by the blowoff velocity, converges to that for the conventional (single tube) piloted burner operated at the same fuel/air ratio.

When fuel is delivered through the central tube or jet $(\mathrm{FJ})$ and air though the annulus, there is an optimum recess distance, $L_{\mathrm{r}} \sim 75 \mathrm{~mm}$, that increases the blowoff velocity by roughly 40 percent, as shown in Fig. 7c. This enhancement of flame stability can be attributed to the inhomogeneous mixture fraction profile at the jet exit, which places near-stoichiometric mixtures at the jet boundary adjacent to the flow of combustion products from the pilot flame. Stratifiedpremixed combustion near the flame base augments the stabilizing effect of the pilot. Then, within the first ten jet exit diameters downstream $(x / D=10)$, as the pdf of mixture fraction on the rich side of the flame progressively shifts toward higher values, there is a transition in the mode of combustion from stratified-premixed to diffusion-dominated [35]. 
These global features and details of the scalar structure of these flames, based on line-imaged Raman/Rayleigh/CO-LIF measurements, were presented previously [34,36]. In order to better resolve the scalar structure in the near field and investigate the combustion mode transition in greater detail, a second complete set of experiments was undertaken [36]. A method of spatial oversampling, with $20 \mu \mathrm{m}$ data spacing, and wavelet adaptive thresholding and reconstruction (WATR) [42] was applied, yielding improvements in both measurement precision and effective spatial resolution (roughly $60 \mu \mathrm{m}$ for flame structures oriented near-perpendicular to the laser axis).

Here, we consider results for progress variable from selected streamwise locations in two flames: FJ-Lr300-59 (near-homogeneous jet exit profile) and FJ-Lr75-80 (inhomogeneous jet exit profile), each at 70 percent of the blowoff velocity for the respective recess distance. These two conditions are marked by solid symbols in Fig. 7c. The numbers 59 and 80 refer to the bulk exit velocity $(\mathrm{m} / \mathrm{s})$ of each flame. The objectives in presenting results from these turbulent flames are: first, to introduce the behavior of the normalized progress variable in two turbulent flames that have very different scalar structure in the near field; second, to provide an initial look at joint density-weighted statistics of mixture fraction and progress variable; and third, to compare the noise characteristics of the different candidate definitions of progress variable. The potential to measure instantaneous dissipation terms for mixture fraction, progress variable, and the cross dissipation is also briefly discussed. Detailed analysis of the evolution of $(Z, c)$ statistics and the three scalar dissipation terms through the region of combustion mode transition into the region of local extinction in the series of jet flames is beyond the scope of the present paper.

\subsection{Temperature scatter plots and Z,c histograms}

Temperature scatter plots are commonly used to convey information from Raman/Rayleigh experiments in turbulent partially-premixed flames that have incomplete combustion for some fraction of measured samples due to local extinction or flame liftoff, and to compare such results with simulations [e.g., 32-36, 43]. A broad view of the behavior of the normalized progress variable, as compared to temperature, in the two piloted flames is given by Fig. 8, which compares scatter plots of temperature vs. mixture fraction and histograms of joint $(Z, c)$ probability. Data from the positive side of the radial traverse are used. Scatter plots include 
every fifth sample, while all samples are represented in the histograms. (Joint distributions at specific locations will be considered in the next subsection.) Flammability limits are shown as vertical dashed (red) lines on either side of the dashed (black) line marking $Z_{\mathrm{st}}=0.055$ in the frames for FJ-Lr75-80, $x / D=1$. Calculated profiles of $T$ and $c_{O}^{*} / c_{O, e q}$ from laminar flames with $\phi_{\mathrm{r}}=1.3,1.7,2.1$, and 4.8 at a strain rate of $a=100 \mathrm{~s}^{-1}$ are included with the measured results at $x / D=5$ for both flames.

At $x / D=1$, both flames are strongly influenced by the pilot, and there is no indication of local extinction. As for the laminar flames in Fig. 1, progress variable shows a dip near stoichiometric, and the trajectory for lean samples follows a slight arc, remaining close to unity. In the near-homogenous flame FJ-Lr300-59 at $x / D=1,5$ there is a near-linear relationship between progress variable and mixture fraction on the fuel-rich side, and the measurements align well with the laminar flame curve for $\phi_{\mathrm{r}}=4.8$. Conditional fluctuations in $c_{O}$ are small, although the color scale, which includes four orders of magnitude in relative probability, highlights the wings of the distribution in a more obvious way than do the scatter plots. With the onset of local extinction in this flame $(x / D \geq 12)$, the distribution in $c_{O}$ at a given $Z$ becomes much broader. The population of samples near the stoichiometric mixture fraction extends down nearly to $c_{O} / c_{O, e q}=0.2$, and the conditional pdf, $P\left(c_{o} / c_{o, e q} \mid Z=Z_{s t}\right)$, becomes bimodal, as there is a region of reduced probability in the middle of the joint distribution, especially at $x / D=20$ in flame FJ-Lr300-59. This bimodality can also be seen in the temperature scatter plot at the same location, and this observation is qualitatively consistent with conditional pdfs of measured species mass fractions [44] and predicted temperatures [45] in Sandia piloted $\mathrm{CH}_{4} /$ air flame F.

The near-field structure is very different for the flame with the inhomogeneous inflow profile of mixture fraction (FJ-Lr75-80), and at $x / D=1$ the progress variable transitions from 0 to 1 mainly within the flammability limits, indicating stratified-premixed combustion, as discussed in detail in [35]. Moving downstream but before there is significant local extinction, this inhomogeneous flame is characterized by much broader conditional distributions in progress variable on the fuel-rich side compared to the more conventional flame, FJ-Lr300-59. Measured values of $c_{O}$ in fuel-rich mixtures at $x / D=5$ in the inhomogeneous turbulent flame are spread broadly across the trajectories of the laminar flame calculations. Flame FJ-Lr75-80 shows less local extinction at $x / D=12$ than FJ-Lr300-59, even though the exit velocity is higher. This is 
due to the stabilizing effect of the pilot flame being augmented by stratified-premixed combustion in the near field. By $x / D=20$ the distributions of $T$ and $c_{O}$ begin to look more similar in the two flames, as turbulent mixing gradually overcomes the difference in mixture fraction profiles at the jet exit. Broadly speaking, the distributions of $c_{O}$, whether plotted as color-coded histograms or as scatter plots (not shown), provide a view of scalar structure that is comparable to temperature scatter plots. However, the $c_{O}$ results carry additional quantitative information on relative progress of reaction for every measured sample.

\subsection{Joint pointwise statistics}

Figure 9 shows radial profiles of Favre average mixture fraction and progress variable, along with their variances and covariance (the correlation coefficient is considered later), calculated as:

$$
\begin{aligned}
& \tilde{Z}=\overline{\rho Z} / \bar{\rho}, \\
& \widetilde{c_{O}}=\overline{\rho c_{O}} / \bar{\rho}, \\
& \widetilde{Z^{\prime \prime 2}}=\overline{\rho(Z-\tilde{Z})^{2}} / \bar{\rho}, \\
& \widetilde{{c_{O}}^{\prime 2}}=\overline{\rho\left(c_{O}-\widetilde{c_{O}}\right)^{2}} / \bar{\rho}, \\
& \widetilde{Z^{\prime \prime} c_{O}}=\overline{\rho(Z-\tilde{Z})\left(c_{O}-\widetilde{c_{O}}\right)} / \bar{\rho}, \\
& R_{Z C}=\widetilde{Z^{\prime \prime} c_{O} "} /\left(\widetilde{Z^{\prime \prime 2}} \widetilde{c_{O}{ }^{\prime 2}}\right)^{1 / 2} .
\end{aligned}
$$

The same downstream locations as in Fig. 8 are represented. The near field profiles of the scalar variances in the two flames are very different. At $x / D=1$ the peaks in $\widetilde{Z^{12}}$ and $\widetilde{c_{O}}{ }^{12}$ are aligned in flame FJ-Lr300-59, both occurring in the middle of the reacting shear layer between the jet and the pilot. However, in the optimally stabilized flame, FJ-Lr75-80, the peaks in $\widetilde{Z^{\prime \prime 2}}$ occur within the cold interior of the inhomogeneous jet, while peaks in $\widetilde{c_{O}{ }^{12}}$ occur in the center of the reaction zone. Consequently, the magnitude of the covariance $\widetilde{Z^{\prime \prime} c_{O}}$ is much smaller in the inhomogeneous flame than in the more conventional near-homogeneous flame at this first measurement location. The peaks in $\widetilde{Z^{12}}$ and $\widetilde{c_{O}{ }^{12}}$ remain separated at $x / D=5$ in the inhomogeneous flame, and the maximum magnitude in $\widetilde{Z^{\prime \prime} c_{O}}$ in the FJ-Lr75-80 flame remains below half that in the FJ-Lr300-59 flame. By $x / D=12$ in the inhomogeneous case, the peak in $\widetilde{Z^{\prime \prime 2}}$ has moved to the jet centerline, still well separated from the peak in $\widetilde{c_{O}{ }^{12}}$. However, because the radial gradient in mixture fraction is still greater in flame FJ-Lr75-80 across fuel-rich conditions and through the reaction zone, as compared to flame FJ-Lr300-59, the peak in $\overline{Z^{\prime \prime} c_{O}}$ 
now exceeds that in the initially near-homogeneous case. At $x / D=20$ differences remain, but the scalar profiles across the two flames are gradually becoming more similar.

A complementary view of the differences between these two flames is provided in Fig. 10 by mapping the radial coordinate onto the mixture fraction coordinate and plotting $\widetilde{c_{O}}, \widetilde{Z " c_{O}}$, and the correlation coefficient from Eq. (10), $R_{Z c}$ against $\tilde{Z}$. Note first that at $x / D=1$ in both flames, the correlation coefficient spikes to 0.85 at the stoichiometric condition. This is an experimental artifact resulting from the fact that gradients in $\tilde{Z}$ and $\widetilde{c_{O}}$ are near zero across the pilot products, and fluctuations in both $Z$ and $c_{O}$ are due mainly to noise in the measurement of $Y_{\mathrm{O} 2}$. At $x / D=5$ in flame FJ-Lr75-80, the correlation coefficient still reaches 0.57 at the stoichiometric mixture fraction, as highlighted by the corresponding arrowhead in Fig. 10. However, at the same location in flame FJ-Lr300-59, the correlation coefficient has dropped to -0.55 , nearly in line with neighboring values for leaner and richer mixtures. This decay of the spike in $R_{Z c}$ near stoichiometry is an indicator of the entrainment of pilot products into the jet. Comparing the curves of $R_{Z c}$ in the two flames at $x / D=5$, it is clear that the pilot products are entrained more rapidly in flame FJ-Lr300-59, even though the jet velocity is lower. This can be attributed to the fact that heat release from stratified-premixed combustion in the near field of flame FJ-Lr75-80 suppresses the growth rate of the turbulent shear layer and the entrainment rate of pilot products.

As might be expected from Fig. 9, the correlation coefficient in the FJ-Lr300-59 flame at $x / D=1,5$ is close to -1 across most of the fuel rich part of the radial profiles due to the nearlinear dependence of progress variable on mixture fraction across this region. This dependence is near-linear because chemical reaction rates are low and changes in progress variable are determined primarily by mixing. Because the peaks in $\widetilde{Z^{12}}$ and $\widetilde{c_{O}{ }^{2}}$ are misaligned and the magnitude of the covariance is much smaller in the near field of flame FJ-Lr75-80, the magnitude of the correlation coefficient, $R_{Z c}$, is also smaller. At $x / D=1$ in this flame, the correlation coefficient in the middle of the stratified-premixed reaction zone is roughly -0.2. This relatively weak, but still negative correlation reflects the mixture fraction distribution in the jet fluid at the edge of the reaction zone, which from Figs. 8 and 9 can be seen to be slightly rich on average. If the recess distance were gradually decreased from $L_{\mathrm{r}}=75 \mathrm{~mm}$, one would expect the correlation coefficient to pass through zero and then become positive as the distribution of mixture fraction samples at the edge of the jet evolved toward being lean on average, while still 
being back-supported by the stoichiometric pilot products. However, the magnitude of $R_{Z c}$ in the main heat release region of the reaction zone in flame FJ-Lr75-80 increases rapidly with streamwise distance as the mixture fraction distribution at the edge of jet becomes richer and the mode of combustion evolves from stratified-premixed toward diffusion-dominated.

It is important to note that the behavior of the correlation coefficient, $R_{Z c}$, depends upon definition of progress variable. Specifically, the behavior of $R_{Z c}$ in fuel-lean regions would be very different if a progress variable without normalization were used. Referring to Fig. 2a, $Y_{O, b}$, the progress variable without normalization, increases monotonically with increasing mixture fraction from zero until reaching its peak value, while $c_{O}$, the normalized progress variable, follows an arch (non-monotonic) across lean conditions. Consequently, one should expect the correlation coefficient for fluctuations in $Z$ and $Y_{O, b}$ to be large and positive across lean regions of the turbulent flames, at least upstream of the onset of local extinction. This highlights the need to use consistent definitions when comparing statistics from experiments and simulations.

At $x / D=5$, still within the near field of this transitioning flame, $R_{Z c}$ reaches -0.7 just on the fuel-rich side of the stoichiometric contour, and $R_{Z c}$ approaches -0.8 slightly farther downstream at $x / D=7$ (not shown) but before the onset of local extinction. This relatively high measured correlation contradicts the common modeling assumption of statistical independence of mixture fraction and progress variable in partially-premixed flames. With the onset of local extinction still farther downstream, the correlation coefficient becomes weaker in both flames. Clearly, the statistical correlation of fluctuations in mixture fraction and progress variable in this type of partially-premixed flame can vary significantly depending on inflow conditions, location within the flame, and the degree of local extinction.

While it is common in flamelet modeling of partially-premixed turbulent combustion to assume statistical independence of mixture fraction and progress variable, there has been recent work to develop flamelet modeling approaches that relax this assumption. Swaminathan and coworkers approximate the joint probability density function, $P(Z, c)$, from the locally modeled means, variances, and covariance of mixture fraction and progress variable, using a copola [911]. Experimental results on such joint statistics are rare [9], so measurements of pointwise or spatially filtered joint pdfs in turbulent partially-premixed flames should prove valuable in the 
further development and validation of such flamelet-based models, as well non-flamelet modeling approaches.

Figure 11 presents joint histograms of mixture fraction and progress variable corresponding to $0.3-\mathrm{mm}$ segments from the radial profiles presented above, with each segment centered at the location where $\tilde{Z}=0.065$. This value of mixture fraction is of interest because it is roughly the value corresponding to peak heat release rate in the more highly strained, partially-premixed laminar flame calculations considered in Section 3. At $x / D=1$ the distributions in the two turbulent piloted flames are very different, with the inhomogeneous FJ-Lr75-80 case having a much broader distribution in progress variable. Each frame in Fig 11 lists first and second moments, as well as the correlation coefficient for the measured population. The biggest differences are in the mean and variance of progress variable, with $\widetilde{c_{O}{ }^{12}}$ in the inhomogeneous case being more than two orders of magnitude greater. The correlation coefficient is relatively low in magnitude at this location in both flames. In the near-homogeneous case $R_{Z c}=-0.138$ because the reaction zone is not very turbulent at $x / D=1$, allowing for a narrow mixture fraction distribution that is centered on a value for which the gradient in mean progress variable is small. There is a highly correlated fuel-rich tail of the distribution that extends toward the cold jet values, but the population in this tail is a small fraction of the total. The mixture fraction variance in flame FJ-L575-80 is larger by a factor of 6 due to incomplete mixing upstream of the exit, and the variance in progress variable is larger by a factor of more than 100 due to the stratified-premixed mode of combustion at this location. However, as noted above, the correlation coefficient is relatively low at -0.2 due to the near vertical orientation of the distribution.

At $x / D=5$ the magnitude of the correlation coefficient is much greater in both flames; -0.646 in FJ-Lr75-80 and -0.820 in FJ-Lr300-59. Clearly, an assumption of statistical independence would be inconsistent with the observed structure in both flames. Moving further downstream the distributions in the two flames become more similar. A broad range in mixture fraction is sampled in both. However, at $x / D=12, \widetilde{Z^{\prime \prime 2}}$ in flame FJ-Lr75-80 is still only half that in the more conventional FJ-Lr-300 flame. As discussed in [35] the probability of local extinction reaches a maximum farther downstream for the inhomogeneous case, FJ-Lr75-80, due to the 
additional heat release from stratified-premixed combustion in the near field and lower levels of scalar dissipation. This difference is also reflected the joint histograms in Fig. 11.

\subsection{Comparison of measurement precision}

In evaluating alternative definitions of progress variable, it is important to consider experimental noise, as well as systematic errors due to fluorescence interference or inconsistent normalization. Measurement precision is particularly important in the context of scalar gradients and dissipation (Section 4.4) because noise always increases the measured mean dissipation rate [e.g., 46,47].

Figure 12 compares rms fluctuation levels of $c_{O}, c_{Y}, c_{C}$, and $c_{T}$, the temperature-based progress variable defined in the Introduction, from measurements in uniform combustion products above a premixed flat flame burner for nine different equivalence ratios between 0.82 and 1.3. Each symbol represents data averaged over the central third of the 6-mm probe volume and for 100 laser shots in each flame. The species-based progress variables consistently show greater uncertainty in fuel-rich conditions compared to fuel-lean conditions. This is attributed to the different form of the fully-burnt denominators in Eqn. (1) for rich versus lean equivalence ratios. For rich conditions, uncertainty in the calculated fully-burnt state depends primarily on uncertainty in the $\mathrm{O}_{2}$ mass fraction, whereas for lean conditions uncertainty depends on three species $\left(\mathrm{CO}, \mathrm{H}_{2}\right.$, and $\left.\mathrm{CH}_{4}\right)$ which have essentially independent noise statistics. $c_{O}$ shows somewhat higher uncertainty (slightly above one percent in rich conditions) compared to $c_{Y}$ or $c_{C}$ in each flame. However, the difference is not large, and should not outweigh the advantages discussed above.

The statistics for fluctuations in $c_{T}$ are less well converged. This is because uncertainty in the Rayleigh temperature measurement depends on uncertainty in the measured laser energy for each shot, as well as detector readout noise and photon shot noise, whereas uncertainty in laser energy cancels out of the calculation of mass fractions. Averaging over the central third of the probe volume does not reduce the laser energy contribution to temperature uncertainty. The average of the nine values for $c_{T}$ is $\sim 0.006$, which is comparable to the uncertainties in the species based progress variables on the lean side. $c_{T}$ has lower noise than the species-based

progress variables in rich cases, and this could prove important in the context of scalar 
dissipation measurements because high dissipation at slightly fuel-rich conditions is expected to lead to local extinction.

Conditional mean and rms curves for the four normalized progress variables, measured in the near-homogeneous FJ-Lr300-59 flame at $x / D=1$, are shown in Fig. 13. This location near the flame base has no local extinction, and conditional mean curves for $c_{O}, c_{Y}$, and $c_{C}$ are similar to those from the laminar flames at the strained laminar flames with $\phi_{\mathrm{r}}=4.8$ (Figs. 1c and 1h), which corresponds to the overall fuel-air ratio in the jet. Here, the correction to remove the cusp at $Z_{\mathrm{st}}$ has not been applied. As in the laminar flat flames, there is relatively little difference in conditional fluctuations of $c_{O}, c_{Y}$, and $c_{C}$. At $x / D=1$ in fuel-lean mixtures the rms fluctuations are below one percent. These measurements are from the laminar mixing layer between pilot products and coflowing air. Fluctuation levels increase to roughly 4 percent for $Z<0.1$. It is expected that some of the fluctuation on the rich side is due to mixture fraction fluctuations in the jet fluid, which is not perfectly homogeneous at the jet exit even when the central tube recess distance is $300 \mathrm{~mm}$. It is also expected that variations in turbulent strain contribute to the measured fluctuations in progress variable. $c_{C}$ has somewhat higher fluctuations for $Z>0.12$, and $c_{O}$ has slightly higher fluctuations for $Z<0.1$ Measurements of noise in steady laminar partially-premixed flames are planned to further clarify the precision and accuracy of the measurements of progress variable. However, the available evidence suggests that there is no significant noise disadvantage associated with using the oxygen based progress variable, compared to the other two species-based definitions.

Normalization of the temperature progress variable by the equilibrium state is appropriate for the premixed flat flames considered above because there is no ambiguity regarding transport and heat losses are relatively minor. (Temperatures from CARS measurements in these flames gave an average deficit of $35 \mathrm{~K} \pm 30 \mathrm{~K}$ below adiabatic equilibrium.) However, normalization of $c_{T}$ remains a problem for laminar or turbulent partially-premixed flames, where differential diffusion alters both composition and temperature of reacted mixtures. For the purpose of illustration, the reference state for normalization of $c_{T}$ has been taken as the adiabatic equilibrium temperature up to $Z=0.065$ followed by a simple linear decrease to room temperature at $Z=1$. At the upstream location $x / D=1$, lean mixtures are sampled from the laminar mixing layer between the stoichiometric pilot products and coflow air. Because of 
differential diffusion across the laminar mixing layer, the relation between temperature and mixture fraction differs from the equilibrium relationship, and the curve for $c_{T}$ is distorted compared to the species-based progress variables. There are significantly lower fluctuations in $c_{T}$ for rich conditions compared to the species-based progress variables, which again may prove important for scalar dissipation measurements. However, the temperature-based progress variable shows no clear advantage in precision for conditions near stoichiometric, and noise in $c_{T}$ increases rapidly moving toward zero mixture fraction. This results from the fact that the measured mixture fraction is used to determine the equilibrium reference temperature, and the effect of uncertainty in the measured mixture fraction is increasingly amplified as the denominator $\left(T_{e q}(Z)-T_{0}\right)$ in the $c_{T}$ expression becomes small. It should be noted that the normalized species-base progress variables are expected to exhibit very large uncertainty approaching $Z=1$ in a pure diffusion flame because both numerator and denominator in the calculation (e.g., Eqn. 1b) approach zero.

\subsection{Potential to measure dissipation terms}

As mentioned in the Introduction, normalization of a progress variable introduces a dependence on mixture fraction that leads to additional terms in its transport equation. These terms include dissipation of mixture fraction, dissipation of progress variable, and the cross dissipation. Measurements of the 1D (radial) contribution to mixture fraction dissipation were reported by Cutcher et al. [35] for all five of the flames initially reported in [35], including the two cases considered here. These dissipation results were based on the higher-resolution, wavelet-denoised measurements, and it was shown that they were fully resolved in all hightemperature regions of the flame, including the near-field reaction zones. Preliminary results suggest that it may also be possible to measure 1D contributions to progress variable dissipation and the cross dissipation. Crossed planar imaging of OH LIF was also applied in these experiments to allow determination of the instantaneous flame orientation. Estimation of all of the 3D dissipation terms from their 1D projections is problematic because it is not known whether the flame normal determined from $\mathrm{OH}$ isocontours is aligned with the normal vectors for progress variable and/or mixture fraction. These issues will be addressed in future work. 


\section{Conclusions}

The main objective of this work was to identify a definition of progress variable that could be calculated from major species measured by Raman/Rayleigh laser diagnostics in partiallypremixed methane flames. Several possible definitions of a normalized progress variable were considered, including two based on the sums of product mass fractions, $Y_{\mathrm{CO} 2}+Y_{\mathrm{CO}}$ and $Y_{\mathrm{CO} 2}+Y_{\mathrm{CO}}+Y_{\mathrm{H} 2 \mathrm{O}}+Y_{\mathrm{H} 2}$. A new progress variable, $c_{O}$, was introduced and defined as the mass of oxygen bound in the main products $\left(\mathrm{CO}_{2}, \mathrm{H}_{2} \mathrm{O}\right.$ and $\left.\mathrm{CO}\right)$ divided by the mass of oxygen that would be bound if the sample were brought to its fully-burnt state by converting deficient reactants to $\mathrm{CO}_{2}$ and $\mathrm{H}_{2} \mathrm{O}$. Laminar flame calculations were used to compare the behavior of the candidate progress variables, and differences were determined to be relatively small. The effects of using the fully-burnt state for normalization rather than equilibrium were considered. It was argued that the oxygen based progress variable, normalized by the fully-burnt state offered advantages, including avoidance of any assumption regarding molecular transport. It was shown that inclusion of a larger number of species in calculating $c_{O}$ (18 versus 7) made negligible difference, such that the seven major species accessible to Raman/Rayleigh laser diagnostics are sufficient to quantify reaction progress in partially-premixed methane flames at atmospheric pressure. A simple correction was proposed to remove the slope discontinuity at the stoichiometric mixture fraction, which results from normalization by the fully-burnt state. The proposed progress variable can be calculated easily from simulation results as a post-processing step, independent of the modeling approach, allowing for consistent comparisons.

The measured joint statistics of mixture fraction, $Z$, and progress variable, $c_{O}$, were presented for two of the Sydney piloted partially-premixed methane-air jet flames, that have significantly different mixture fraction profiles at the jet exit. Favre average radial profiles of first and second moments and the correlation coefficient, $R_{Z, c}$, highlighted differences in near-field scalar structure between the two flames. Examples of joint $Z, c_{O}$ histograms, sampled from 0.3-mm segments centered at the location where $\tilde{Z}=0.065$ in each radial profile, were presented. This mixture fraction value corresponds roughly to the location of peak heat release rate on the fuelrich side in the laminar flame calculations. The common modeling assumption of statistical independence of mixture fraction and progress variable was clearly contradicted by the measurements. 


\section{Acknowledgments}

The authors acknowledge informative and insightful discussions with many members of the theory and modeling community, especially Forman Williams, Matthias Ihme, and Nedunchezhian Swaminathan. Work at Sandia was supported by the Division of Chemical Sciences, Geosciences and Biosciences, Office of Basic Energy Sciences, US Department of Energy. Sandia National Laboratories is a multiprogram laboratory operated by Sandia Corporation, a Lockheed Martin Company, for the United States Department of Energy under contract DE-AC04-94-AL85000. Work at the University of Sydney was supported by the Australian Research Council.

\section{References}

[1] P. Domingo, L. Vervisch, K. Bray, Partially premixed flamelets in LES of nonpremixed turbulent combustion, Combust. Theory Model. 6 (2002) 529-551.

[2] B. Fiorina, R. Baron, O. Gicquel, D. Thevenin, S. Carpentier, N. Darabiha, Modelling non-adiabatic partially premixed flames using flame-prolongation of ILDM, Combust. Theory Model. 7 (2003) 449-470.

[3] C.D. Pierce, P. Moin, Progress-variable approach for large-eddy simulation of non-premixed turbulent combustion, J. Fluid Mech. 504 (2004) 73-97.

[4] B. Fiorina, O. Gicquel, L. Vervisch, S. Carpentier, N. Darabiha, Combust. Flame 140 (2005) 147160.

[5] K. Bray, P. Domingo, L. Vervisch, Role of the progress variable in models for partially premixed turbulent combustion, Combust. Flame 141 (2005) 431-437.

[6] P. Domingo, L. Vervisch, D. Veynante, Large-eddy simulation of a lifted methane jet flame in a vitiated coflow, Combust. Flame 152 (2008) 415-432.

[7] A.W. Vreman, B.A. Albrecht, J.A. van Oijen, L.P.H. de Goey, R.J.M. Bastiaans, Premixed and nonpremixed generated manifolds in large-eddy simulation of Sandia flame D and F, Combust. Flame 153 (2008) 394-416.

[8] M. Ihme, Y.C. See, Prediction of autoignition in a lifted methane/air flame using an unsteady flamelet/progress variable model, Combust. Flame 157 (2010) 1850-1862.

[9] O.R. Darbyshire, N. Swaminathan, A presumed joint pdf model for turbulent combustion with varying equivalence ratio, Combust. Theory Model. 184 (2012) 2036-2067.

[10] S. Ruan, N. Swaminathan, O. Darbyshire, Modelling of turbulent lifted jet flames using flamelets: a priori assessment and a posteriori validation, Combust. Theory Model. 18 (2014) 295-329. 
[11] Z. Chen, S. Ruan, N. Swaminathan, Simulation of turbulent lifted methane jet flames: Effects of airdilution and transient flame propagation, Combust. Flame 162 (2015) 703-716.

[12] R.W. Bilger, S.H. Stårner, R.J. Kee, On reduced mechanisms for methane/air combustion in nonpremixed flames, Combust. Flame 80 (1990) 135-149.

[13] R.S. Barlow, Laser diagnostics and their interplay with computations to understand turbulent combustion, Proc. Combust. Inst. 31 (2007) 49-75.

[14] R.S. Barlow, M.J. Dunn, M.S. Sweeney, S. Hochgreb, Effects of preferential transport in turbulent bluff-body-stabilized lean premixed CH4/air flames, Combust. Flame 159 (2012) 2563-2575.

[15] A. Sevault, M. Dunn, R.S. Barlow, M. Ditaranto, On the structure of the near field of oxy-fuel jet flames using Raman/Rayleigh laser diagnostics, Combust. Flame 159 (2012) 3342-3352.

[16] G. Kuenne, F. Seffrin, F. Fuest, T. Stahler, A. Ketelheun, D. Geyer, J. Janicka, A. Dreizler, Experimental and numerical analysis of a lean premixed stratified burner using 1D Raman/Rayleigh scattering and large eddy simulation, Combust. Flame 159 (2012) 2669-2689.

[17] S. Nambully, P. Domingo, V. Moureau, L. Vervisch, A filtered-laminar-flame PDF sub-grid scale closure for LES of premixed turbulent flames. Part I: Formalism and application to a bluff-body burner with differential diffusion, Combust. Flame 161 (2014) 1756-1774.

[18] F. Proch, A.M. Kempf, Numerical analysis of the Cambridge stratified flame series using artificial thickened flame LES with tabulated premixed flame chemistry, Combust. Flame 161 (2014) 26272646.

[19] M. Ihme, H. Pitsch, Prediction of extinction and reignition in nonpremixed turbulent flames using a flamelet/progress variable model 2. Application in LES of Sandia flames D and E, Combust. Flame 155 (2008) 90-107.

[20] J.A. van Oijen, L.P.H. de Goey, Modelling of premixed counterflow flames using the flameletgenerated manifold method, Combust. Theory Model. 6 (2002) 463-478.

[21] W.J.S. Ramaekers, J.A. van Oijen, L.P.H. de Goey, Apriori testing of flamelet generated manifolds for turbulent partially premixed methane/air flames, Flow Turbulence Combust. 84 (2010) 439-458.

[22] M. Ihme, L. Shunn, J, Zhang, Regularization of reaction progress variable for application to flamelet-based computational models, J. Comp. Phys. 231 (2012) 7715-7721.

[23] A. Najafi-Yazdi, B. Cuenot, L. Mongeau, Systematic definition of progress variables and Intrinsically Low-Dimensional, Flamelet Generated Manifolds for chemistry tabulation, Combust. Flame 159 (2012) 1197-1204.

[24] Y.S. Niu, L. Vervisch, P.D. Tao, An optimization-based approach to detailed chemistry tabulation: Automated progress variable definition, Combust. Flame (2013) 776-785. 
[25] U. Prüfert, S. Hartl, F. Hunger, D. Messig, M. Eiermann, C. Hasse, Flow Turbulence Combust. 94 (2015) 593-617.

[26] M. Ihme, H. Wu, Progress variable and combustion regime: Relevance to modeling, in R.S. Barlow ed., TNF13 Proceedings, http://www.sandia.gov/TNF/13thWorkshop/TNF13.html.

[27] M.S. Sweeney, S. Hochgreb, M.J. Dunn, R.S. Barlow, The structure of turbulent stratified and premixed methane/air flames I: Non-swirling flows, Combust. Flame 159 (2012) 2896-2911.

[28] M.M. Kamal, R.S. Barlow, S. Hochgreb, Conditional analysis of turbulent premixed and stratified flames on local equivalence ratio and progress of reaction, Combust. Flame 162 (2015) 3896-3913.

[29] Y.C. Chen, R.W. Bilger, Experimental investigation of three-dimensional flame-front structure in premixed turbulent combustion-I: hydrocarbon/air bunsen flames, Combust. Flame 131 (2002) 400-435.

[30] G. Magnotti, R.S. Barlow, Effects of high shear on the structure and thickness of turbulent premixed methane/air flames stabilized on a bluff-body burner, Combust. Flame 162 (2015) 100-114.

[31] A. Parente, J.C. Sutherland, L. Tognotti, P.J. Smith, Identification of low-dimensional manifolds in turbulent flames, Proc. Combust. Inst. 32 (2009) 1579-1586.

[32] A.R. Masri, R.W. Dibble, R.S. Barlow, The structure of turbulent nonpremixed flames revealed by Raman-Rayleigh-LIF measurements, Prog. Energy Combust. Sci. 22 (1996) 307-362.

[33] J. Xu, S.B. Pope, Pdf calculations of turbulent nonpremixed flames with local extinction, Combust. Flame 123 (2000) 281-307.

[34] S. Meares, V.N. Prasad, G. Magnotti, R.S. Barlow, A.R. Masri, Stabilization of piloted turbulent flames with inhomogeneous inlets, Proc. Combust. Inst. 35 (2015) 1477-1484.

[35] R.S. Barlow, S. Meares, G. Magnotti, H. Cutcher, A.R. Masri, Local extinction and near-field structure in piloted turbulent $\mathrm{CH}_{4}$ /air jet flames with inhomogeneous inlets, Combust. Flame 162 (2015) 3516-3540.

[36] H. Cutcher, G. Magnotti, R. S. Barlow, and A. R. Masri, Turbulent flames with compositionally inhomogeneous inlets: resolved measurements of scalar dissipation rates," Proc. Combust. Inst. (in press).

[37] CHEMKIN-PRO 15131, Reaction Design: San Diego, 2013.

[38] G.P. Smith, D.M. Golden, M. Frenklach, N.W. Moriarty, B. Eiteneer, M. Goldenberg, C.T. Bowman, R.K. Hanson, S. Song, W.C. Gardiner, Jr., V.V. Lissianski, Z. Qin, http://www.me.berkeley.edu/gri_mech/GRI-Mech 3.0, (2015).

[39] R.S. Barlow, A.N. Karpetis, J.H. Frank, J.-Y. Chen, Scalar profiles and NO formation in laminar opposed-flow partially premixed methane/air flames, Combust. Flame 127 (2001) 2102-2118. 
[40] G. Magnotti, U. KC, P.L. Varghese, R.S. Barlow, Raman spectra of methane, ethylene, ethane, dimethyl ether, formaldehyde and propane for combustion applications, JQSRT 163 (2015) 80-101.

[41] G. Magnotti, R.S. Barlow, Dual-resolution Raman spectroscopy for measurements of temperature and twelve species in hydrocarbon-air flames, Proc. Combust. Inst. (in press).

[42] M.S. Sweeney, S. Hochgreb, M.J. Dunn, R.S. Barlow, Multiply conditioned analyses of stratification in highly swirling methane/air flames, Combust. Flame 160 (2013) 322-334.

[43] C.M. Arndt, M. Severin, C. Dem, M. Stöhr, A.M. Steinberg, W. Meier, Experimental analysis of thermos-acoustic instabilities in a generic gas turbine combustor by phase-correlated PIV, chemiluminescence, and laser Raman scattering measurements, Exp Fluids (2015) 56-96.

[44] R.S. Barlow, J.H. Frank, Effects of turbulence on species mass fractions in methane/air jet flames, Proc. Combust. Inst. 27 (1998) 1087-1095.

[45] R.P. Lindstedt, S.A. Louloudi, E.M. Vaos, Joint scalar probability density function modeling of pollutant formation in piloted turbulent jet diffusion flames with comprehensive chemistry, Proc. Combust. Inst. 28 (2000) 149-156.

[46] S. P. Nandula, T. M. Brown, and R. W. Pitz, "Measurements of scalar dissipation in the reaction zones of turbulent nonpremixed H2 air flames," Combust. Flame 99 (1994) 775-783.

[47] G.-H. Wang, N.T. Clemens, P.L. Varghese, Two-point, high-repetition-rate Rayleigh thermometry in flames: techniques to correct for apparent dissipation induced by noise, Appl. Opt. 44 (2005) 67416751. 


\section{Figure captions:}

Fig. 1. Behavior of three progress variables normalized by the fully-burnt state in laminar opposed-flow $\mathrm{CH}_{4}$ air flames with fuel-side equivalence ratios of 1.3, 3.2, 4.8, 9.5, and inf. (pure $\mathrm{CH}_{4}$ ).

Fig. 2. a) Comparison of the three progress variables with normalization by the fully-burnt state, FB, and the equilibrium state, EQ. Each plot also compares the numerators (non-normalized versions of each progress variable; blue, dot-dash), and the denominators corresponding to fully-burnt normalization (black, solid) and equilibrium normalization (red, dash).

Fig. 3. Illustration of differences among the three progress variables when equilibrium is used as the normalization reference for a laminar flame calculation that includes multi-component transport.

Fig. 4. Renormalization to eliminate the slope discontinuity in $c_{O}$. Solid (black) curves for $c_{O}$ correspond to the flames in Fig. $1 \mathrm{~b}, \mathrm{~g}\left(\phi_{\mathrm{r}}=3.2\right)$. The dashed (red) curve, $c_{O, e q}$, is obtained by applying Eqn. (1) to the equilibrium table. Note that the maximum deviation of $c_{O, e q}$ from unity is only 3 percent. The smooth dot-dash (blue) curve is calculated as the ratio $c_{O} / c_{O, e q}$.

Fig. 5. Comparison of mixture fraction and oxygen-based progress variable calculated from mass fractions of 18 species $\left(Z^{*}, c_{O}^{*}\right)$ versus seven species $\left(Z, c_{O}\right)$ in three laminar flames.

Fig. 6. Temperature, heat release rate, and progress variable in laminar opposed-flow flames with seven different rich-side equivalence ratios, each calculated for two strain rates as labeled. The two (red) symbols along each curve mark locations of lean and rich local maxima in heat release rate.

Fig. 7. a) Cutaway schematic of the burner with dimensions in $\mathrm{mm}$; b) isometric solid model; c) dependence of blowoff velocity on recess distance for the FJ configuration with fuel in the central jet. The solid symbols show conditions for the FJ-Lr300-59 (black square) and FJ-Lr75-80 (blue circle).

Fig. 8. a) Scatter plots of temperature versus mixture fraction, including data from the $(+)$ side of the radial profile at each axial location. The stoichiometric mixture fraction $\left(Z_{\mathrm{st}}=0.055\right)$ is marked by a dashed vertical line in each frame. Flammability limits are indicated by the vertical dashed (red) lines in the lower left frame. The solid (red) curves in the $x / D=5$ frames are from laminar flame calculations with rich-side equivalence ratios of $\phi_{\mathrm{r}}=1.3$, $1.7,2.1$, and 4.8. b) joint histograms of progress variable and mixture fraction corresponding to the same data as shown in the temperature scatter plots. The color scale covers 4 orders of magnitude and is scaled by the maximum probability occurring in each plot.

Fig. 9. Radial profiles of Favre mean (left), variances, and covariance (right) of mixture fraction and progress variable in the two turbulent flames. 
Fig. 10. Favre average half-profiles $(r / D \geq 0)$ of $\widetilde{c_{O}}, \widetilde{Z^{\prime \prime} c_{O}}$, and $R_{Z c}$ plotted against Favre average mixture fraction, $\tilde{Z}$, from the same radial profile.

Fig 11. Histograms of joint probability of mixture fraction and progress variable from $0.3-\mathrm{mm}$ width segments centered at the radial location corresponding to $\tilde{Z}=0.065$. Color scale as in Fig. 8. The radial location, mean progress variable, second moments, and the correlation coefficient, $R_{Z c}$ are listed in each frame.

Fig. 12. Comparison of rms fluctuations in progress variables measured in uniform combustion products above a premixed $\mathrm{CH} 4 /$ air flat flame operated at different equivalence ratios.

Fig. 13. Conditional mean and rms of four normalized progress variables measured at $x / D=1$ in the nearhomogeneous flame, FJ-Lr300-59. 

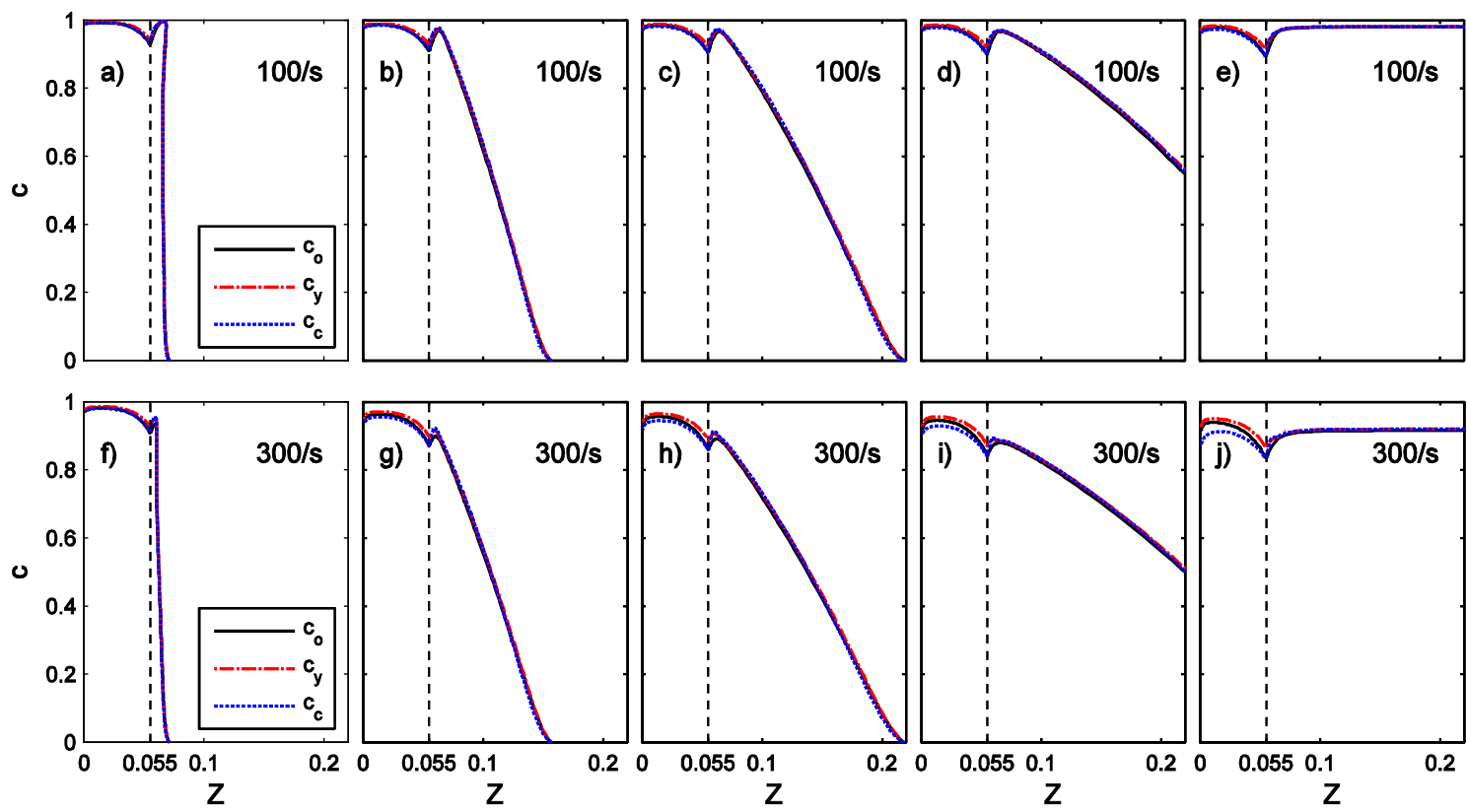

Fig. 1. 

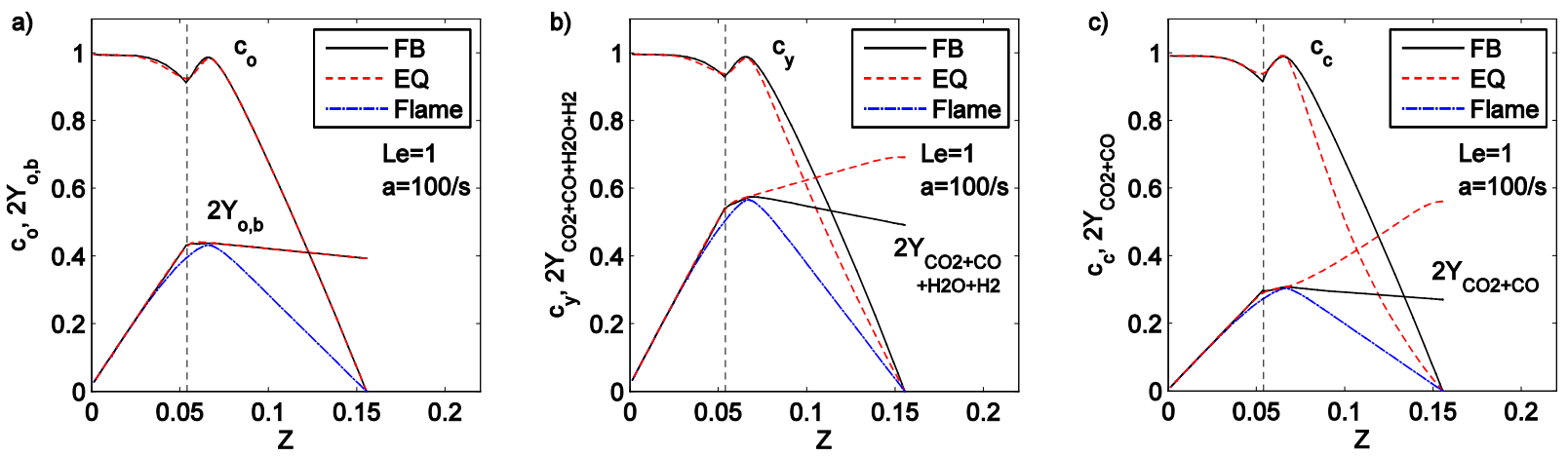

Fig. 2a, b, c 


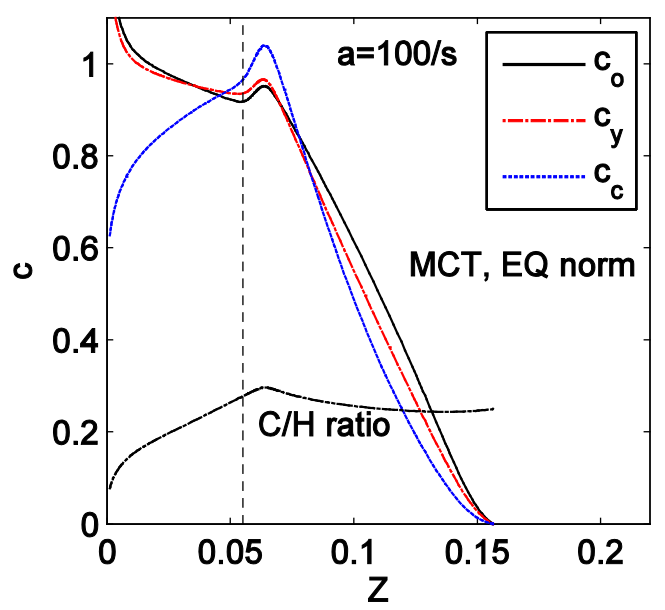

Fig. 3. 


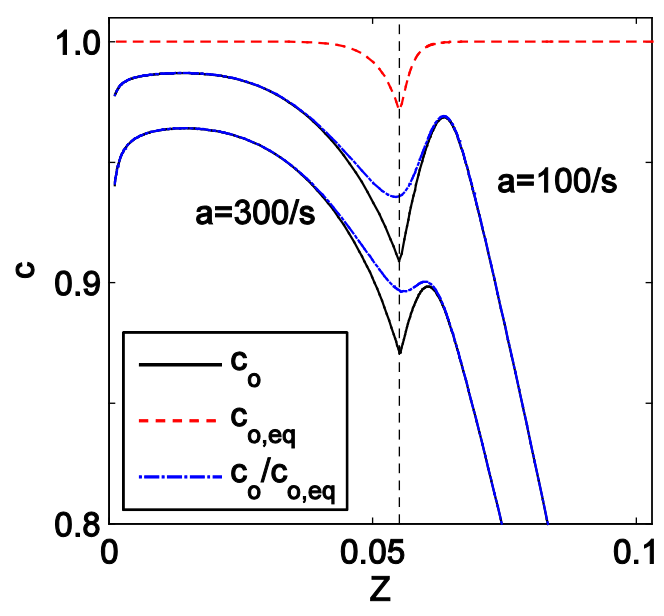

Fig. 4. 

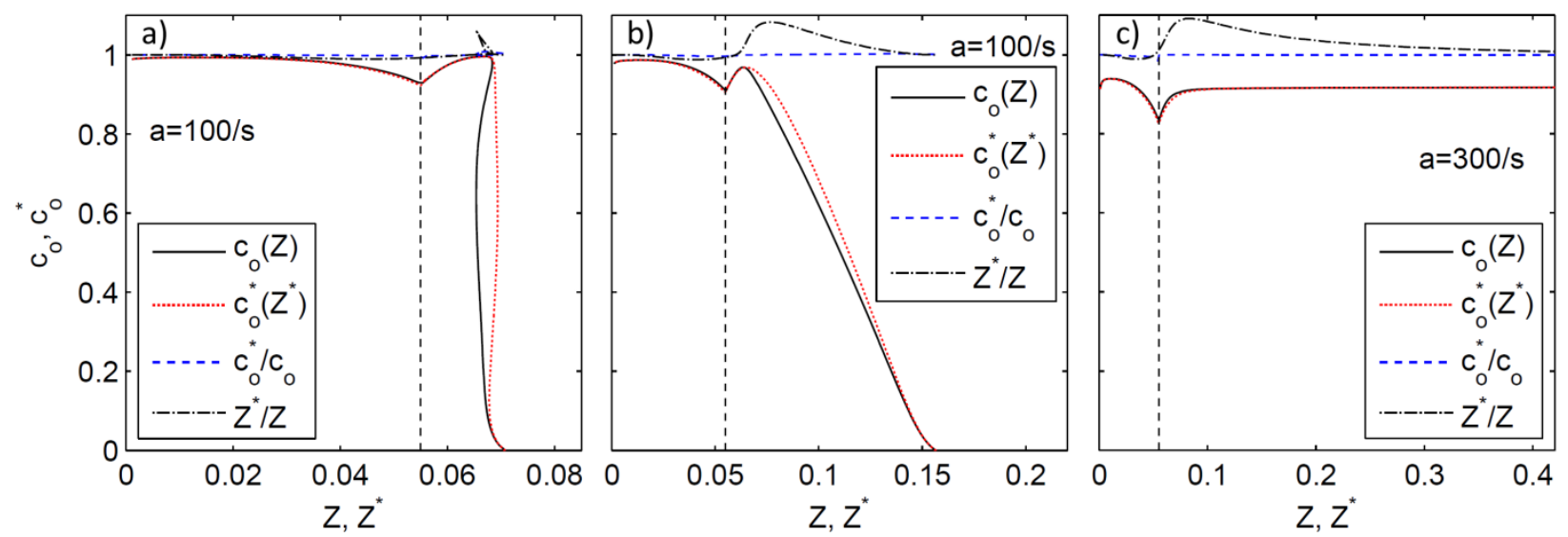

Fig. 5. 

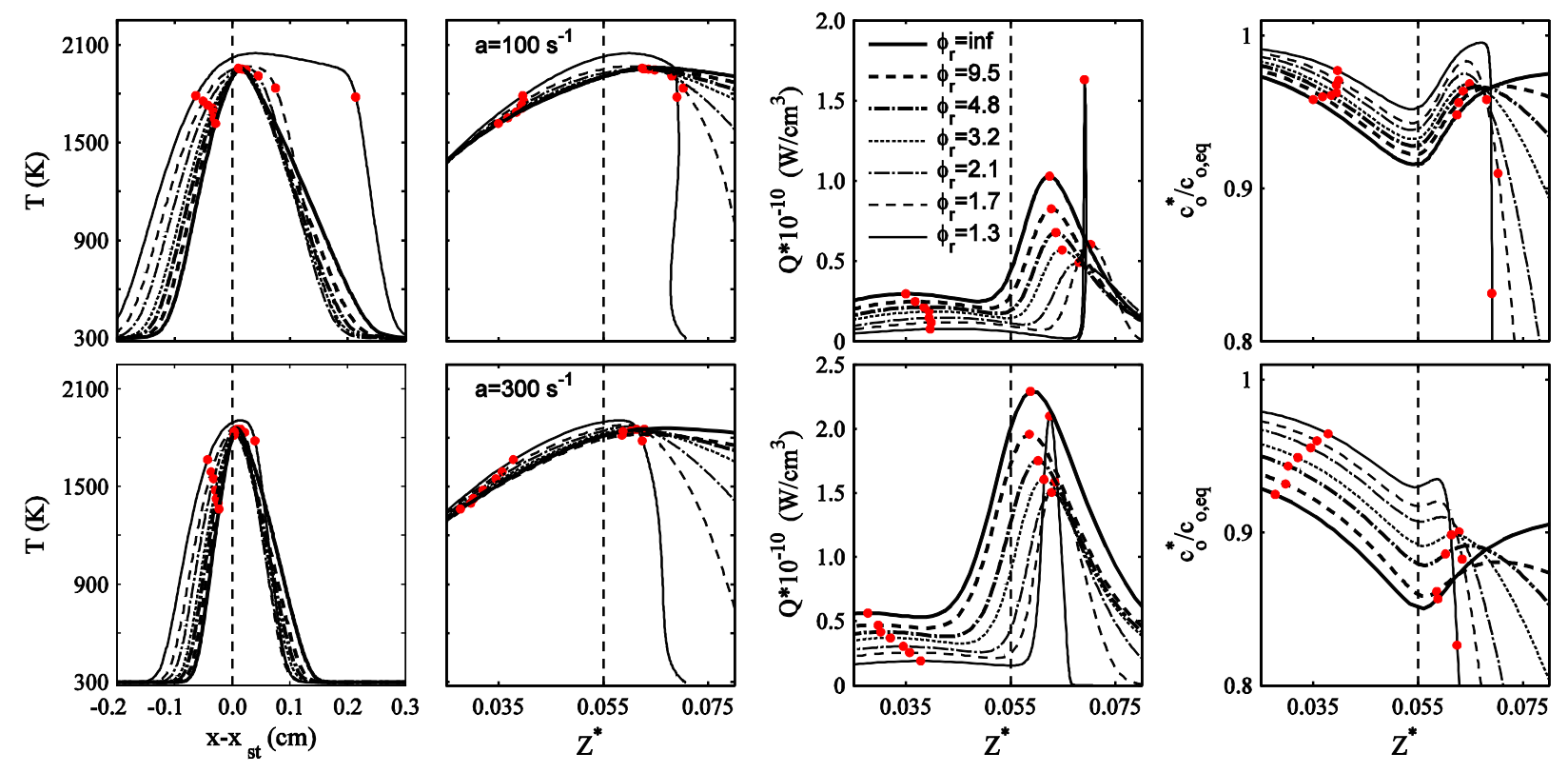

Fig. 6. 


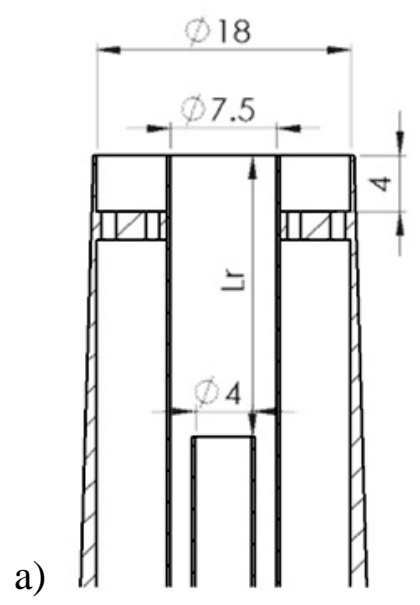

b)
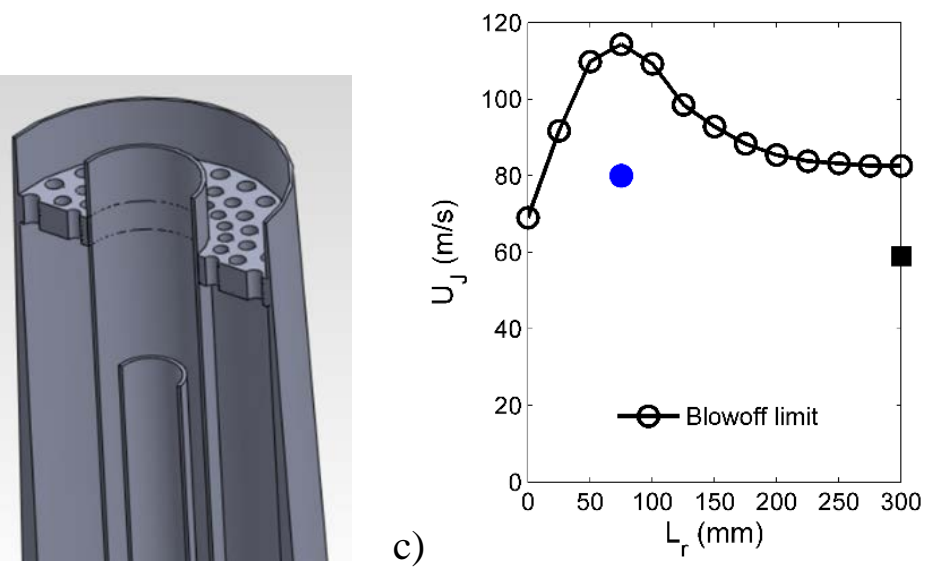

Fig. 7a, b, c. 

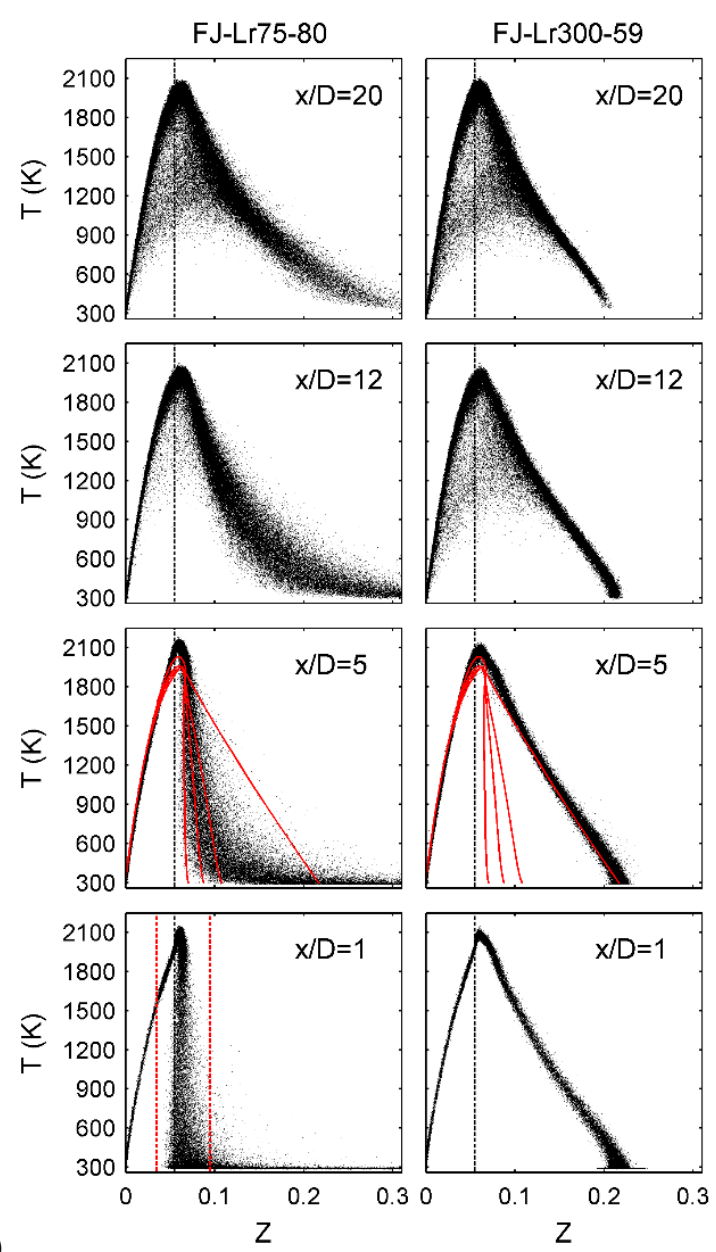

a)

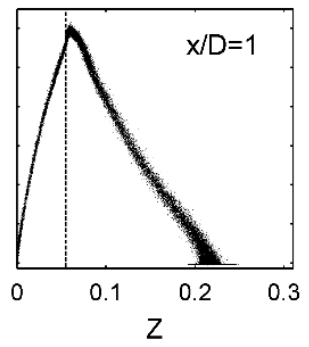

Fig. 8a, b
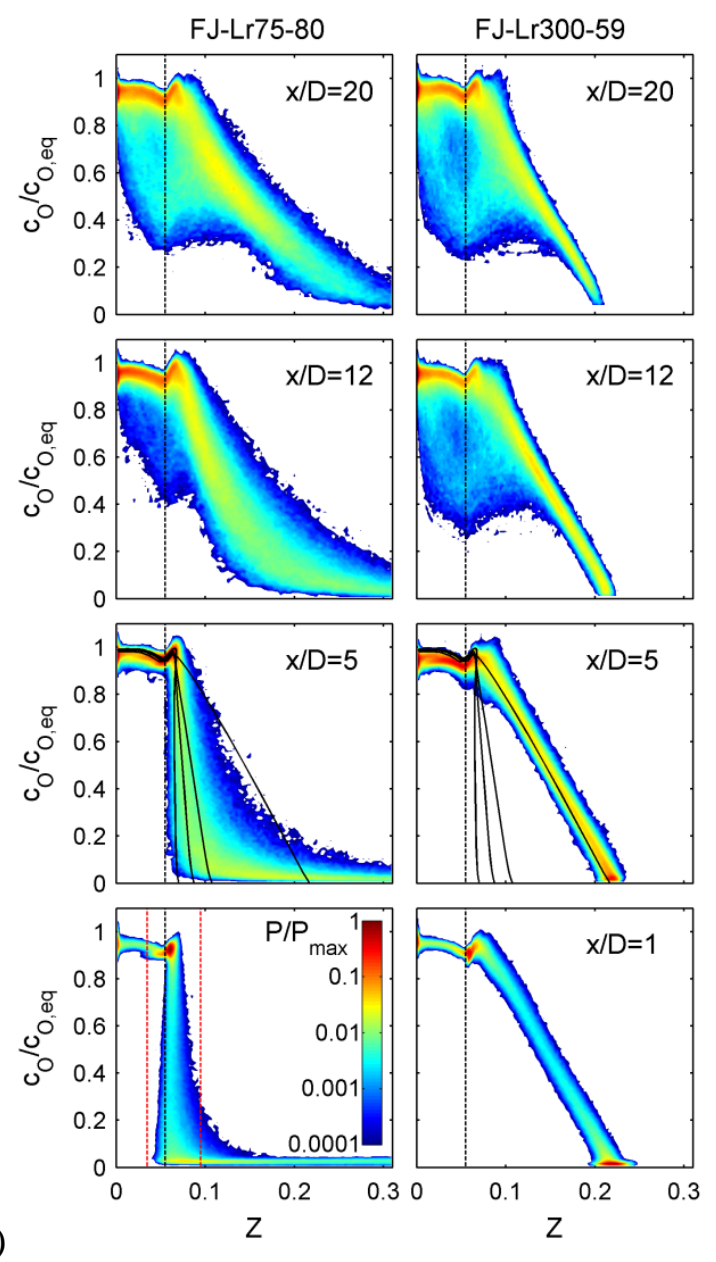

b) 

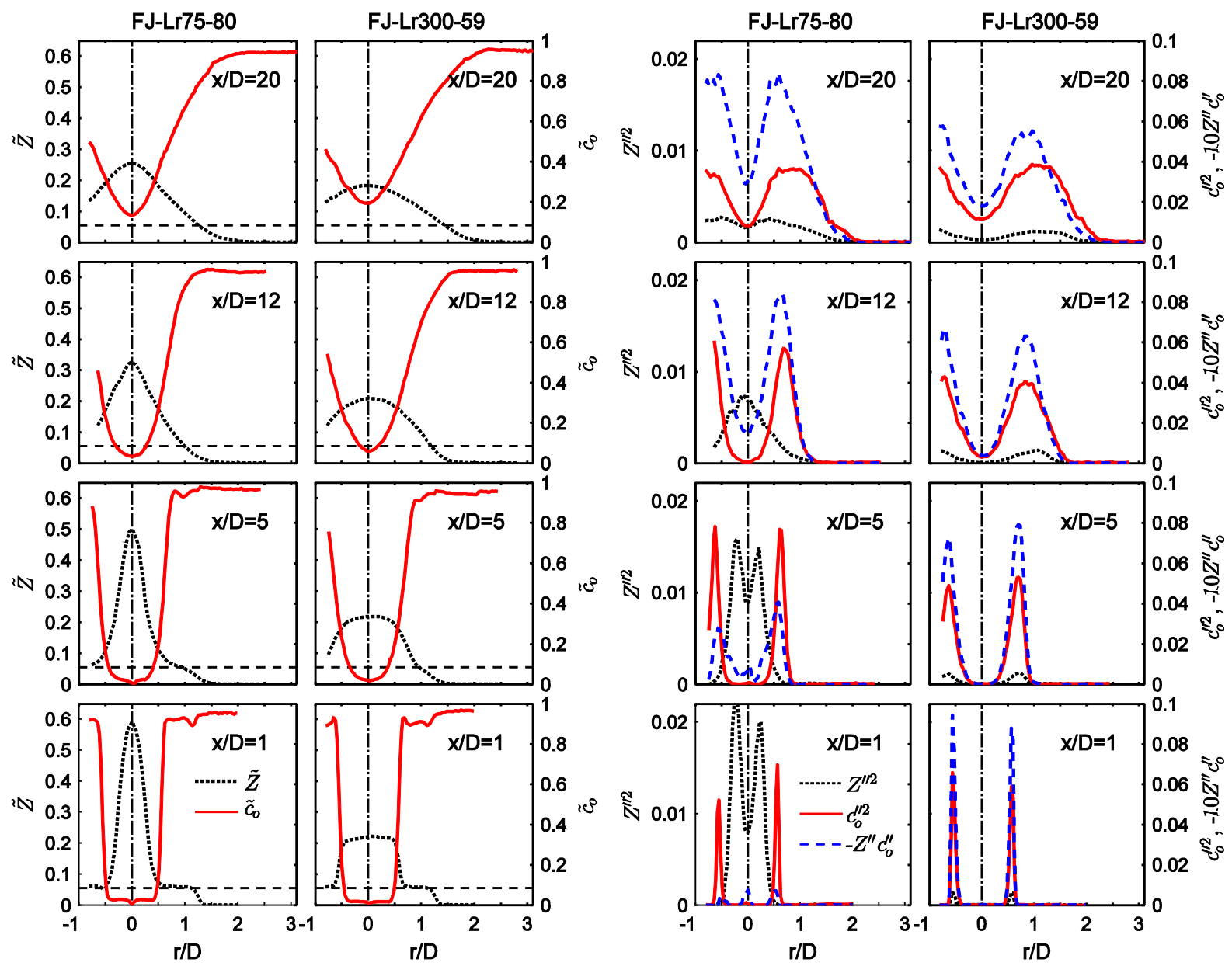

Fig. 9. 


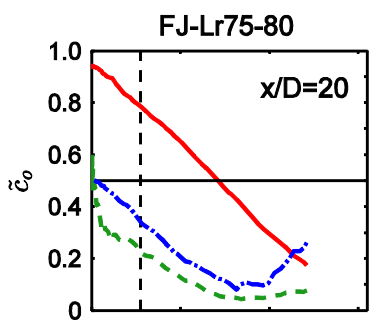

FJ-Lr300-59
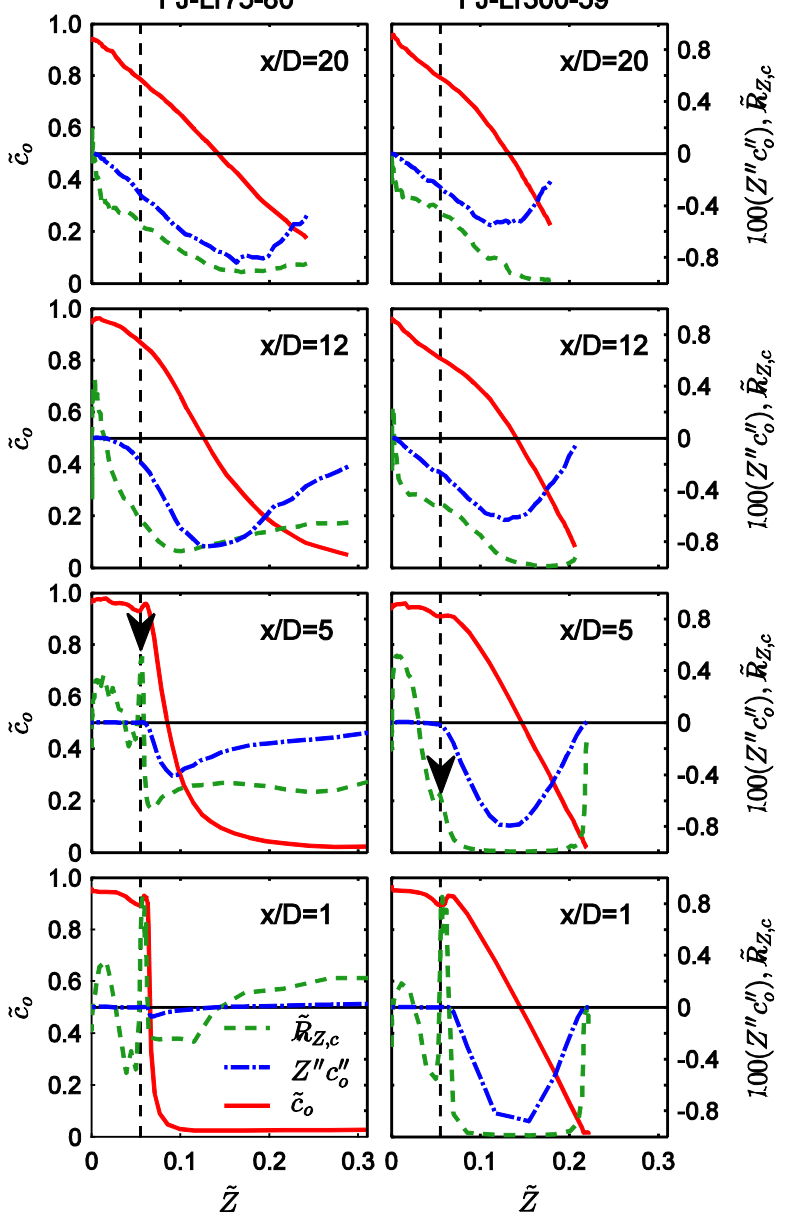

Fig. 10. 

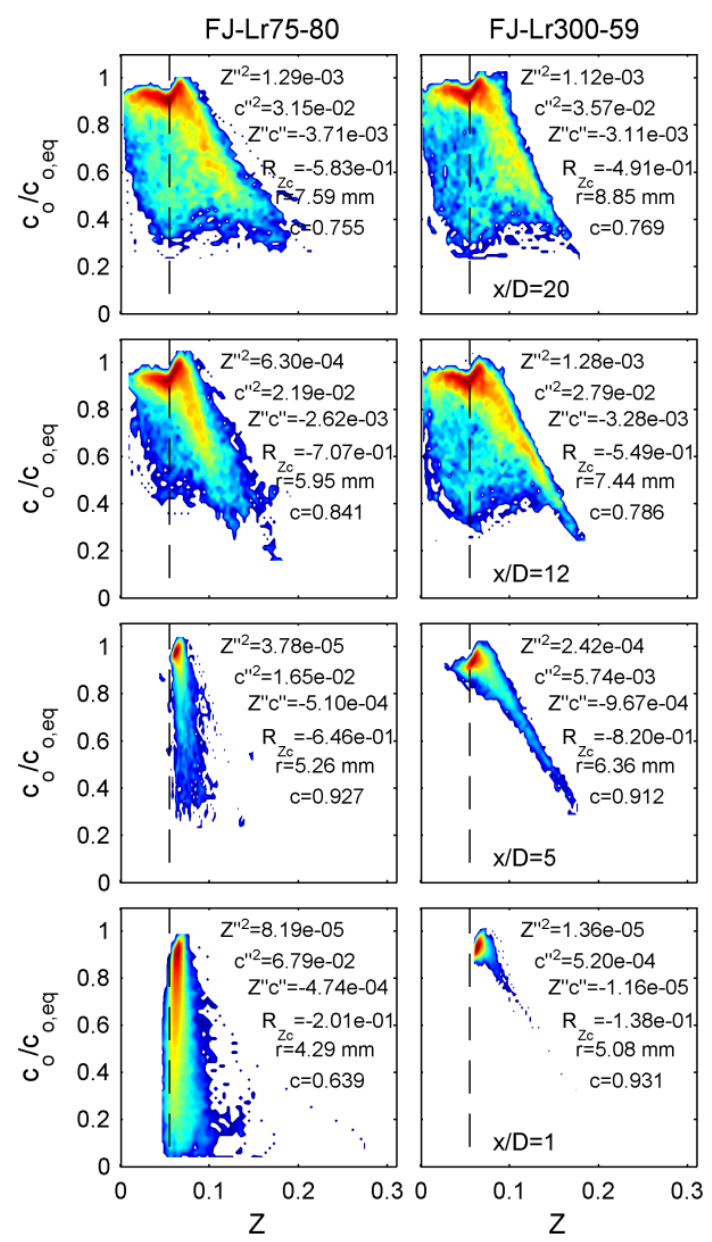

Fig 11. 


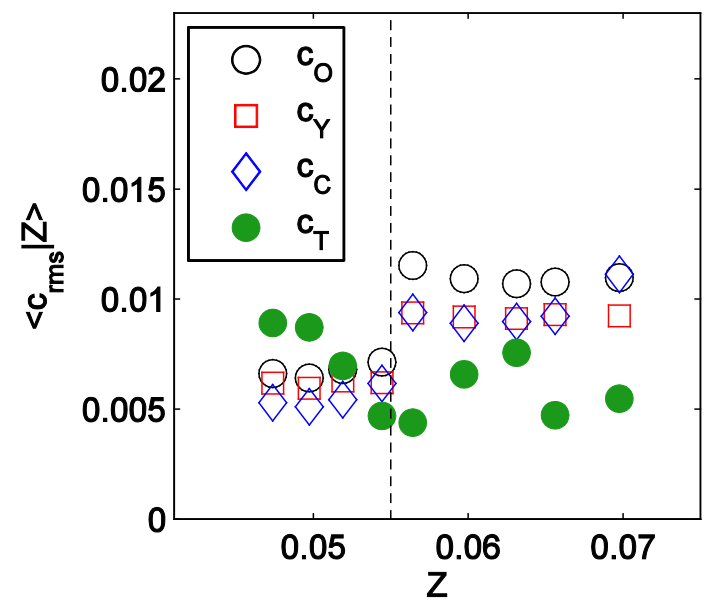

Fig. 12. 

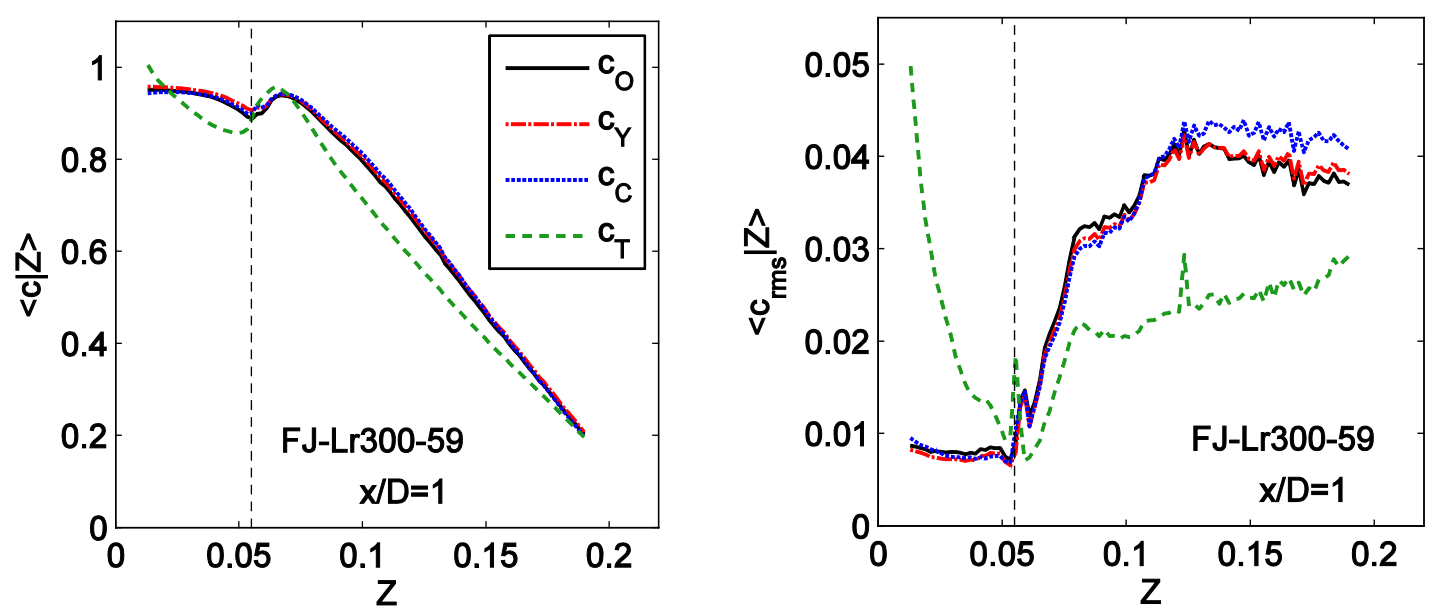

Fig. 13. 